\title{
The Transient Responses of an Axisymmetric Tropical Cyclone to Instantaneous Surface Roughening and Drying $\mathscr{A}$
}

\author{
Jie Chen AND Daniel R. Chavas
}

Purdue University, West Lafayette, Indiana

(Manuscript received 20 November 2019, in final form 15 May 2020)

\begin{abstract}
Inland tropical cyclone (TC) impacts due to high winds and rainfall-induced flooding depend strongly on the evolution of the wind field and precipitation distribution after landfall. However, research has yet to test the detailed response of a mature TC and its hazards to changes in surface forcing in idealized settings. This work tests the transient responses of an idealized hurricane to instantaneous transitions in two key surface properties associated with landfall: roughening and drying. Simplified axisymmetric numerical modeling experiments are performed in which the surface drag coefficient and evaporative fraction are each systematically modified beneath a mature hurricane. Surface drying stabilizes the eyewall and consequently weakens the overturning circulation, thereby reducing inward angular momentum transport that slowly decays the wind field only within the inner core. In contrast, surface roughening initially $(\sim 12 \mathrm{~h})$ rapidly weakens the entire low-level wind field and enhances the overturning circulation dynamically despite the concurrent thermodynamic stabilization of the eyewall; thereafter the storm gradually decays, similar to drying. As a result, total precipitation temporarily increases with roughening but uniformly decreases with drying. Storm size decreases monotonically and rapidly with surface roughening, whereas the radius of maximum wind can increase with moderate surface drying. Overall, this work provides a mechanistic foundation for understanding the inland evolution of real storms in nature.
\end{abstract}

\section{Introduction}

While the majority of existing tropical cyclone (TC) research has focused on its coastal impacts, TCs also induce significant inland hazards, especially damaging wind and rainfall-induced flooding. Economic loss can be significant due to agricultural impacts, power outages, and infrastructure damage associated with strong winds (Berg 2009; Cangialosi et al. 2018) and inland freshwater flooding (Villarini et al. 2014). From 1970 to $1999,63 \%$ of all TC related deaths were inland deaths (Rappaport 2000). Among the 2325 individual deaths in the United States from 1963 to 2012, rainfall-induced flood deaths occurred more than any other hazard, whereas $5 \%-10 \%$ of the deaths were caused by nontornadic winds (Rappaport 2014). For example, the slow-moving Hurricanes Harvey (2017) and Florence (2018) induced catastrophic inland flooding over Texas

\footnotetext{
Supplemental information related to this paper is available at the Journals Online website: https://doi.org/10.1175/JAS-D-190320.s1.
}

Corresponding author: Jie Chen, chen2340@purdue.edu and the Carolinas, respectively (Blake and Zelinsky 2018). Moreover, rainfall production is intimately linked to the TC low-level wind field (Lu et al. 2018). Therefore, credible estimates of inland damage risk depend on an understanding of how the TC wind and precipitation fields evolve after landfall. This is increasingly important given that storms may be moving more slowly on average (Kossin 2018), a trend that is expected to continue in a future climate (Emanuel 2017). However, the responses of a mature TC and associated hazards to the physical processes that underlie landfall have not been systematically tested in an idealized setting and remain poorly understood. This lack of knowledge greatly inhibits our ability to predict inland hazards and estimate hazard risk in both operations and long-term risk assessment.

Research has analyzed TC landfall using historical data, climate models, and real-world landfall simulations (Powell 1987; Powell et al. 1991; Davis et al. 2008;

\footnotetext{
Publisher's Note: This article was revised on 4 August 2020 to fix a production error that resulted in the inclusion of an equation, Eq. (12), that should have been deleted prior to publication. The remaining equations have been renumbered appropriately.
} 
Jian and Wu 2008; Kruk et al. 2010; Lin et al. 2010; Murakami et al. 2016), and empirical models exist to predict the postlandfall decay of storm intensity (Tuleya 1994; Kaplan and DeMaria 1995). Though these studies are essential for analyzing individual case studies and for directly estimating real-world risk, the complexity of the real world makes it difficult for such simulations to provide a fundamental understanding of the response of a storm to changes in surface properties associated with landfall. A limited number of idealized studies or sensitivity tests exist, though they focus primarily on the evolution of storm intensity (Tuleya and Kurihara 1975, 1978; Kishtawal et al. 2012), or the overall asymmetric wind distribution (Wong and Chan 2007), and they are ripe for updating given advances in theory or numerical modeling since their publication. A few studies have applied theoretical models to examine the postlandfall response of the boundary layer (Powell 1982; Kepert 2012) and the precipitation distribution associated with real-world storms (Kepert 2013; Lu et al. 2018). However, systematic, idealized experiments testing the response of the tropical cyclone wind and precipitation distributions to landfall have yet to be performed.

Meanwhile, decades of research have advanced our understanding of tropical cyclones over an ocean surface that may also be useful for understanding the postlandfall evolution (Malkus and Riehl 1960; Ooyama 1964, 1969; Anthes 1974; Emanuel 1986). In particular, TC potential intensity theory (Emanuel 1986) provides a natural starting point for understanding the response to landfall. This theory idealizes a mature, steady-state tropical cyclone as a Carnot heat engine, in which entropy fluxes from the ocean surface are used to maintain the circulation against surface frictional dissipation. It has also been extended to predict time-dependent changes in storm intensity including the effects of ventilation of environmental air by wind shear (Tang and Emanuel 2010, 2012). This theory predicts the maximum potential intensity that a storm may achieve in a given thermodynamic environment as

$$
V_{p}=\sqrt{\frac{C_{k}}{C_{d}} \eta(\Delta k)},
$$

where

$$
\begin{aligned}
\eta & =\frac{T_{\mathrm{SST}}-T_{\mathrm{tpp}}}{T_{\mathrm{tpp}}}, \\
\Delta k & =C_{p}\left(T_{\mathrm{SST}}-T_{a}\right)+L_{v}\left[q^{*}\left(T_{\mathrm{SST}}\right)-q_{a}\left(T_{a}\right)\right],
\end{aligned}
$$

$C_{k}$ and $C_{d}$ are bulk exchange coefficient for surface enthalpy and momentum, respectively, $\Delta k$ is the difference between the saturation enthalpy of the ocean surface and the enthalpy of the overlying near-surface air, $T_{\mathrm{SST}}$ is the sea surface temperature, $T_{a}$ is the temperature of air overlying the ocean surface, $T_{\mathrm{tpp}}$ is the tropopause temperature, $L_{v}$ is the enthalpy of vaporization, $C_{p}$ is the specific heat capacity of air, $q^{*}$ is the saturation mixing ratio of the ocean surface at the local surface pressure, and $q_{a}$ is the mixing ratio of air overlying the ocean surface.

Equation (1) predicts that the equilibrium intensity will decrease with higher $C_{d}$ (i.e., a rougher surface) and smaller $\Delta k$ (i.e., a drier surface), both of which are associated with a transition from the ocean to the land surface. Indeed, storm intensity is well known to decay rapidly after landfall in both observations and realworld numerical simulations (Kaplan and DeMaria 1995; Knutson et al. 2015), although there are rare cases in which storms maintain their intensity temporarily after landfall, due perhaps to locally enhanced surface heat fluxes or baroclinic enhancement (Emanuel et al. 2008; Evans et al. 2011). Moreover, the existence of shallow land surface water can reduce the landfall decay rate (Shen et al. 2001). Preceding formal potential intensity theory, Ooyama (1964) first demonstrated the essential role of surface enthalpy fluxes to a mature TC in a numerical model. Moreover, Tuleya and Kurihara (1978) performed idealized tests of the sensitivity of TC intensity to surface roughness and surface evaporation, finding that the suppression of evaporation is the dominant factor for the decay of TC intensity after landfall. However, little follow-up work has been performed to test these responses in the context of modern potential intensity theory or to extend the study to the complete low-level wind field.

In addition, recent work has advanced our understanding of the complete wind field over ocean (Colon 1963; Eliassen 1971; Eliassen and Lystad 1977; Merrill 1984; Holland et al. 2010; Chavas et al. 2016). For tropical cyclones over the ocean, a theoretical model for the complete wind field now exists (Chavas et al. 2015) that combines models for the inner, convecting region wind field (Emanuel and Rotunno 2011) and outer, nonconvecting region wind field (Emanuel 2004). This model can capture the first-order structure and variability of the complete low-level wind field over ocean (Chavas and Lin 2016) and can also explain equilibrium wind field structure across both moist and dry surfaces (Cronin and Chavas 2019). However, this model has yet to be tested for the transient response after landfall.

In terms of rainfall, extensive observational and numerical studies have been performed to understand key aspects of the inland rainfall evolution. Asymmetric rainfall characteristics, including mean rain rate, distribution, and total precipitation, are related to landfalling TC intensity on average (Lonfat et al. 2007; Kimball 2008; Liu et al. 2018), though peak rainfall is not correlated with TC intensity (Deng et al. 2017). Rainfall 
asymmetry is often forced by environmental vertical wind shear, nonuniform surface characteristics, and mesoscale convective activity rather than TC intensity (Rogers et al. 2003; Chan et al. 2004; Chen et al. 2006; Hsu and Kuo 2013; Li and Duan 2013; Li et al. 2014; Meng and Wang 2016). More recently, a physics-based TC rainfall model has been developed for risk analysis to model real-world rainfall risk (Zhu et al. 2013; Emanuel 2017). This model quantifies contributions to rainfall from frictional convergence, topographic uplift, vortex stretching, and baroclinic forcing (Lu et al. 2018). However, research has yet to test the transient precipitation response to landfall and its underlying physical mechanisms in an idealized setting.

This work seeks to fill the above knowledge gaps by systematically testing the response of a mature, axisymmetric hurricane to instantaneous surface roughening and drying in an idealized setting. Specifically, we seek to answer the following research questions in this work:

1) In addition to intensity, how does the structure of the wind and precipitation fields respond to surface roughening or drying?

2) What are the time scales of these responses, and how do they differ between each surface forcing?

3) What is the radial structure of the response, and how does it differ between each surface forcing?

4) Can we understand the physical mechanisms that govern these responses?

To answer the above questions, we perform and analyze two sets of experiments in which the surface beneath an initially quasi-steady hurricane is instantaneously dried or roughened over a range of magnitudes (section 2). We first characterize the basic transient responses of TC intensity, size, and precipitation across all experiments (section 3 ). We then focus on one representative experiment from each set for an in-depth, comparative analysis of the radial structure of the responses (section 4). For these two cases, we further isolate physical processes governing the responses of the low-level wind field via absolute angular momentum budget analysis and of the precipitation responses via a simple dynamic-thermodynamic decomposition (section 5). We summarize the major findings of this work and discuss limitations and avenues for future work (section 6).

\section{Methods}

\section{a. Overview}

As noted above, tropical cyclone landfall is principally associated with two basic surface forcings acting on a mature hurricane: surface roughening and surface drying. Here we seek to analyze the transient responses of the TC vortex and precipitation to these two forcings in idealized experiments in which each forcing is applied instantaneously over a range of magnitudes. We note that, for real-world landfall, inland surface roughness and wetness have significant spatiotemporal variations (Cosby et al. 1984; Stull 1988). Surface drag coefficient can range from 0.002 to 0.3 based on different terrain types (Holmes 2001). Representing such complexity requires sophisticated land surface schemes and boundary layer schemes to parameterize soil, vegetation, and land use (Zhang et al. 2017, 2019; Davis et al. 2008; Nolan et al. 2009; Jin et al. 2010; Kishtawal et al. 2012) that translate to large spatiotemporal variation in surface roughness and wetness that will vary from storm to storm. Simulations with such schemes provide essential insight for simulating the detailed evolution of real-world storms.

Here, though, we seek a more general understanding of the most fundamental surface forcings associated with landfall in a simplified setting absent the pronounced spatiotemporal heterogeneity in surface properties found in the real world. Hence, we focus on experiments testing the TC transient response to surface drying and roughening in axisymmetric geometry with a uniform environment and boundary forcing. Future work may add additional types of complexity to understand the effects that arise from asymmetries in the storm, surface, or environment. This idealized work serves as a rung in the hierarchy of models (Held 2005) that, in conjunction with more complex idealized experiments and real-world simulations, will improve both the basic understanding of landfall and the prediction of its inland hazards.

\section{b. Model description}

Numerical simulation experiments in axisymmetric geometry are performed using the Bryan Cloud Model (CM1), version 19.7 (v19.7). CM1 is suitable for use in a broad range of atmospheric science applications across scales, including hurricanes (Chavas and Emanuel 2014; Peng et al. 2018) and severe thunderstorms (Sherburn and Parker 2019; Trapp et al. 2018). CM1 satisfies nearexact conservation of both mass and energy in a reversible saturated environment (Bryan and Fritsch 2002; Rotunno and Bryan 2012). The model solves the fully compressible equations of motion in height coordinates on an $f$ plane for flow velocities $(u, v, w)$, nondimensional pressure $\pi$, potential temperature $\theta$, and the mixing ratios of water in vapor, liquid, and solid states $q_{x}$ on a fully staggered Arakawa C-type grid.

\section{c. Model setup}

The basic axisymmetric, $f$-plane model setup is similar to Chavas and Emanuel (2014) (Table. 1). The Coriolis parameter is set to $f=5 \times 10^{-5} \mathrm{~s}^{-1}$. The outer wall is set to $L=3000 \mathrm{~km}$ with $3-\mathrm{km}$ radial grid spacing. A stretched grid is used in vertical with a constant grid 
TABLE 1. Model and environment parameter values of the baseline and CTRL simulation.

\begin{tabular}{|c|c|c|c|c|c|}
\hline Model & Name & Value & Environment & Name & Value \\
\hline$l_{h}$ & Horizontal mixing length & $750 \mathrm{~m}$ & $T_{\mathrm{SST}}$ & Sea surface temperature & $300 \mathrm{~K}$ \\
\hline$l_{\text {inf }}$ & Asymptotic vertical mixing length & $100 \mathrm{~m}$ & $\mathrm{~T}_{\mathrm{tpp}}$ & Tropopause temperature & $200 \mathrm{~K}$ \\
\hline$C_{k}$ and $C_{d}$ & $\begin{array}{l}\text { Exchange coefficients of enthalpy } \\
\text { and momentum }\end{array}$ & 0.0015 & $Q_{\text {cool }}$ & $\begin{array}{l}\text { Radiative cooling rate } \\
\quad \text { (potential temperature) }\end{array}$ & $1 \mathrm{~K}_{\text {day }^{-1}}$ \\
\hline$H_{\text {domain }}$ & Height of model top & $25 \mathrm{~km}$ & $f$ & Coriolis parameter & $5 \times 10^{-5} \mathrm{~s}^{-1}$ \\
\hline$L_{\text {domain }}$ & Radius of model outer wall & $3000 \mathrm{~km}$ & & & \\
\hline
\end{tabular}

spacing of $100 \mathrm{~m}$ below $z=3 \mathrm{~km}$, smoothly varying vertical grid spacing from $100 \mathrm{~m}$ at $z=3 \mathrm{~km}$ to $500 \mathrm{~m}$ at $z=12 \mathrm{~km}$, and then constant grid spacing of $500 \mathrm{~m}$ from $z=12$ to $25 \mathrm{~km}$. In axisymmetric geometry, turbulent eddies cannot be resolved directly and thus are parameterized using a modified Smagorinsky-type scheme with distinct mixing lengths in the radial $\left(l_{h}=750 \mathrm{~m}\right.$, constant) and vertical directions (asymptotes to $l_{\text {inf }}=$ $100 \mathrm{~m}$ at $z=\infty$ ). In CM1 v19.7, the horizontal Rayleigh damper (hrdamp $=1$ ) is only applied to the vertical velocity at lateral boundaries, and not to the horizontal velocities, to minimize artificial sources of momentum. The upper-level Rayleigh damping (irdamp = 1) is applied above $z=20 \mathrm{~km}$, damping horizontal and vertical velocities toward the base state. The radiation scheme simply applies a constant cooling rate, $Q_{\text {cool }}$, to the potential temperature where the absolute temperature exceeds a threshold temperature, $T_{\mathrm{tpp}}$, and is otherwise relaxed back to $T_{\mathrm{tpp}}$ :

$$
\frac{\partial \theta}{\partial t}= \begin{cases}\frac{\theta\left(p, T_{\mathrm{tpp}}\right)-\theta(p, T)}{\tau_{\mathrm{strat}}} & T<T_{\mathrm{tpp}} . \\ -Q_{\mathrm{cool}} & T>T_{\mathrm{tpp}}\end{cases}
$$

We set $Q_{\text {cool }}=1 \mathrm{~K} \mathrm{day}^{-1}$ and $T_{\mathrm{tpp}}=200 \mathrm{~K}$, which corresponds to an approximate tropopause temperature. The Newtonian relaxation time scale is $\tau_{\text {strat }}=12 \mathrm{~h}$. This simple approach neglects all water-radiation and temperature-radiation feedbacks. A similar setup applied to absolute temperature is also used in Cronin and Chavas (2019). Surface latent heat fluxes $F_{\mathrm{LH}}$ and sensible heat fluxes $F_{\mathrm{SH}}$ are calculated from the bulk-aerodynamic formulas of surface mixing ratio fluxes $F_{\mathrm{qv}}$ and potential temperature fluxes $F_{\theta}$ in CM1 (sfcphysics.F) as

$$
\begin{aligned}
F_{\mathrm{LH}} & =\rho L_{v} F_{\mathrm{qv}}, \\
F_{\mathrm{SH}} & =\rho C_{p} F_{\theta}, \\
F_{\mathrm{qv}} & =\varepsilon s_{10} C_{q} \Delta q, \quad \text { and } \\
F_{\theta} & =s_{10} C_{h} \Delta \theta,
\end{aligned}
$$

where $C_{q}$ and $C_{h}$ are the exchange coefficients for the surface moisture and sensible heat, respectively, $s_{10}$ is the $10-\mathrm{m}$ wind speed, $\Delta q$ and $\Delta \theta$ are the moisture and heat disequilibrium between the 10-m layer and the sea surface, respectively, and $\varepsilon$ is the surface evaporative fraction ( $\varepsilon=1$ over ocean). Coefficients $C_{q}$ and $C_{h}$ are set equal to each other, and so both represent the single enthalpy exchange coefficient, $C_{k}$. Surface drying is modeled by decreasing $\varepsilon$ to represent the transition from a wet to a drier surface, thus decreasing the surface latent heat fluxes $F_{\mathrm{LH}}$. This representation of surface wetness is slightly simpler than that employed in Cronin and Chavas (2019), which modified the surface saturation mixing ratio.

Surface roughening is modeled by increasing the drag coefficient $C_{d}$, which modifies the surface roughness length $z_{0}$ and, in turn, the friction velocity $u_{*}$ for the surface logarithmic layer in CM1 as

$$
\begin{aligned}
& z_{0}=\frac{z}{\exp \left(\frac{\kappa}{\sqrt{C_{d}}}-1\right)} \text { and } \\
& u_{*}=\max \left[\frac{\kappa s_{1}}{\ln \left(\frac{z_{a}}{z_{0}}+1\right)}, 1.0 \times 10^{-6}\right],
\end{aligned}
$$

where $\kappa$ is the von Kármán constant, $z=10 \mathrm{~m}$ is the reference height, $s_{1}$ is the total wind speed on the lowest model, and $z_{a}$ is approximately equal to the lowest model level height. On the lowest model level, $u_{*}$ determines the subgrid shear stress $\tau_{i j}$ [e.g., $\tau_{23}=u_{*}^{2} v_{1} / s_{1}$, where $(i, j)=(2,3)$ indicates the $v-w$ shear stress], which is used to calculate the turbulent tendencies. For the azimuthal wind, the turbulent tendency is

$$
T_{v}=\frac{1}{\rho}\left(\frac{\partial \tau_{12}}{\partial x}+\frac{\partial \tau_{22}}{\partial y}+\frac{\partial \tau_{23}}{\partial z}\right)
$$

in the azimuthal velocity tendency equation. Hence, instantaneous changes in the parameters $C_{d}$ and $\varepsilon$ are applied to model surface roughening and drying, respectively. 
We note that $\varepsilon$ is specifically applied to the latent fluxes $F_{\mathrm{LH}}$, as surface enthalpy fluxes for mature storms over ocean are dominated by latent heat (Charney and Eliassen 1964; Kuo 1965; Ooyama 1969; Cione et al. 2000; Guimond et al. 2011). Sensible heat fluxes $F_{\mathrm{SH}}$ can become important when latent heat fluxes are very strongly reduced $(\varepsilon<0.1)$, in which case the sensible heating provides a significant fraction of the total surface enthalpy fluxes. This can be understood via Eq. (1) and is discussed in more detail in the appendix. This suggests that land surface properties may become important for weak inland storms over very dry surfaces, as is occasionally observed with real storms (Evans et al. 2011; Kieu 2015; Shen et al. 2001). For our work here, applying $\varepsilon$ to sensible heat fluxes in addition to latent heat fluxes does not materially change our results. A more proper accounting of surface sensible heat fluxes likely requires experiments using a coupled land-atmosphere model that represent soil heat capacity and thermal diffusivity (Emanuel et al. 2008; Kishtawal et al. 2012). Thus, the role of changes in sensible heat fluxes is left for future work.

Using the above setup, we define an initial sounding as the domain-mean state for the final ten days of a 100-day simulation that has reached statistical equilibrium. This approach is commonly used in idealized modeling studies of tropical convection (Wing and Emanuel 2014; Wing et al. 2016; Peng et al. 2018). Given that we begin from a long-run simulation (described below), our results are not sensitive to this choice of the initial state, though, as was found in Chavas and Emanuel (2014).

\section{d. Experimental design}

Before conducting surface roughening and drying experiments, we first create a baseline experiment that produces a tropical cyclone over an ocean-like surface where $C_{d}=0.0015, C_{k}=0.0015$, and $\varepsilon=1$. We run this baseline experiment for 200 days to allow the storm to reach a statistical steady state for at least 50 days (Fig. 1a). We then identify the most stable period during this long-term evolution, here selected as days 150-165; results are not sensitive to this choice of restart time. From this stable period, we define a 10-day control experiment (CTRL) as the ensemble mean of five 10-day segments of the baseline experiment (days 150-160, 151-161, 152-162, 153-163 and 154-164; Fig. 1b). We then perform ensembles of restart experiments with $C_{d}$ or $\varepsilon$ instantaneously modified beneath the stable storm. $C_{d}$ is enhanced by a factor of $2,4,6,8$, and 10 , whereas $\varepsilon$ is reduced from 1 to $0.7,0.5,0.3,0.25$, and 0.1 , representing various levels of surface roughening or drying, respectively. For each roughening or drying experiment, we also run five 10-day experiments restarting from each of the five CTRL ensemble segments. These five runs are then averaged into a 10-day ensemble representing the corresponding experiment. This ensemble approach reduces noise and increases the robustness of the responses. We use $\tau$ to denote the time since the start of a given experiment.

Note that $C_{k}$ may also vary in the real world, although there remain large uncertainties with regard to $C_{k}$ and the ratio $C_{k} / C_{d}$ (Bell et al. 2012). A previous study suggests that the ratio of $C_{k} / C_{d}$ has less effect on the maximum wind speed in numerical simulations than in theoretical models, although the maximum wind speed is still proportional to $C_{k} / C_{d}$ (Bryan 2012). Little is known about its inland variability or how $C_{k}$ might scale with $C_{d}$ over land. For our work we simply hold $C_{k}$ constant, which translates to a decrease in the ratio of $C_{k} / C_{d}$ with roughening. Further discussion of treatment of the ratio of $C_{k} / C_{d}$ is provided in section 6. Our aim is to test the transient response specifically to enhanced $C_{d}$. The transient response of a TC to surface roughening while holding $C_{k} / C_{d}$ fixed is a valuable topic for future work.

\section{e. Analysis}

We first characterize the temporal evolution of the transient responses across all experiments by normalizing the evolution of storm intensity $v_{m}$ (maximum tangential wind speed at lowest model level), size $r_{\max }$ and $r_{34 \mathrm{kt}}$ (radius of maximum tangential wind speed and 34-kt tangential wind, respectively; $1 \mathrm{kt} \approx$ $0.51 \mathrm{~m} \mathrm{~s}^{-1}$ ), and total precipitation $P$ (hourly total rainfall within $r=150 \mathrm{~km}$ ) each by their time-dependent CTRL values.

We use the potential intensity equation [Eq. (1)] to define a theoretical prediction for the equilibrium response in maximum wind speed given by

$$
\frac{V_{p \mathrm{EXP}}}{V_{p \mathrm{CTRL}}}=\frac{\sqrt{\frac{C_{k}}{\left(C_{d}\right)_{\mathrm{EXP}}} \eta\left[C_{p} \Delta T+L_{v}(\Delta q)_{\mathrm{EXP}}\right]}}{\sqrt{\frac{C_{k}}{C_{d}} \eta \Delta k}}
$$

for comparison with the simulated response given by $v_{\mathrm{mEXP}} / v_{\mathrm{mCTRL}}$. The $\left(C_{d}\right)_{\mathrm{EXP}}$ is defined by its experimental value, and $(\Delta q)_{\text {EXP }}$ is defined by multiplying its CTRL value by $\varepsilon$. The value of $V_{\mathrm{pCTRL}}$ is $69.93 \mathrm{~m} \mathrm{~s}^{-1}$, with $\Delta T$ and $\Delta q$ defined from the environment (averaged over $r=1500-2500 \mathrm{~km}$ ) and $66.37 \mathrm{~m} \mathrm{~s}^{-1}$ defined at $r_{\text {max }}$. Both values are smaller than the actual CTRL $v_{m}$ on the lowest model level $(50 \mathrm{~m})$ of $90.4 \mathrm{~m} \mathrm{~s}^{-1}$. Past work has shown that $v_{m}$ can exceed the potential intensity 

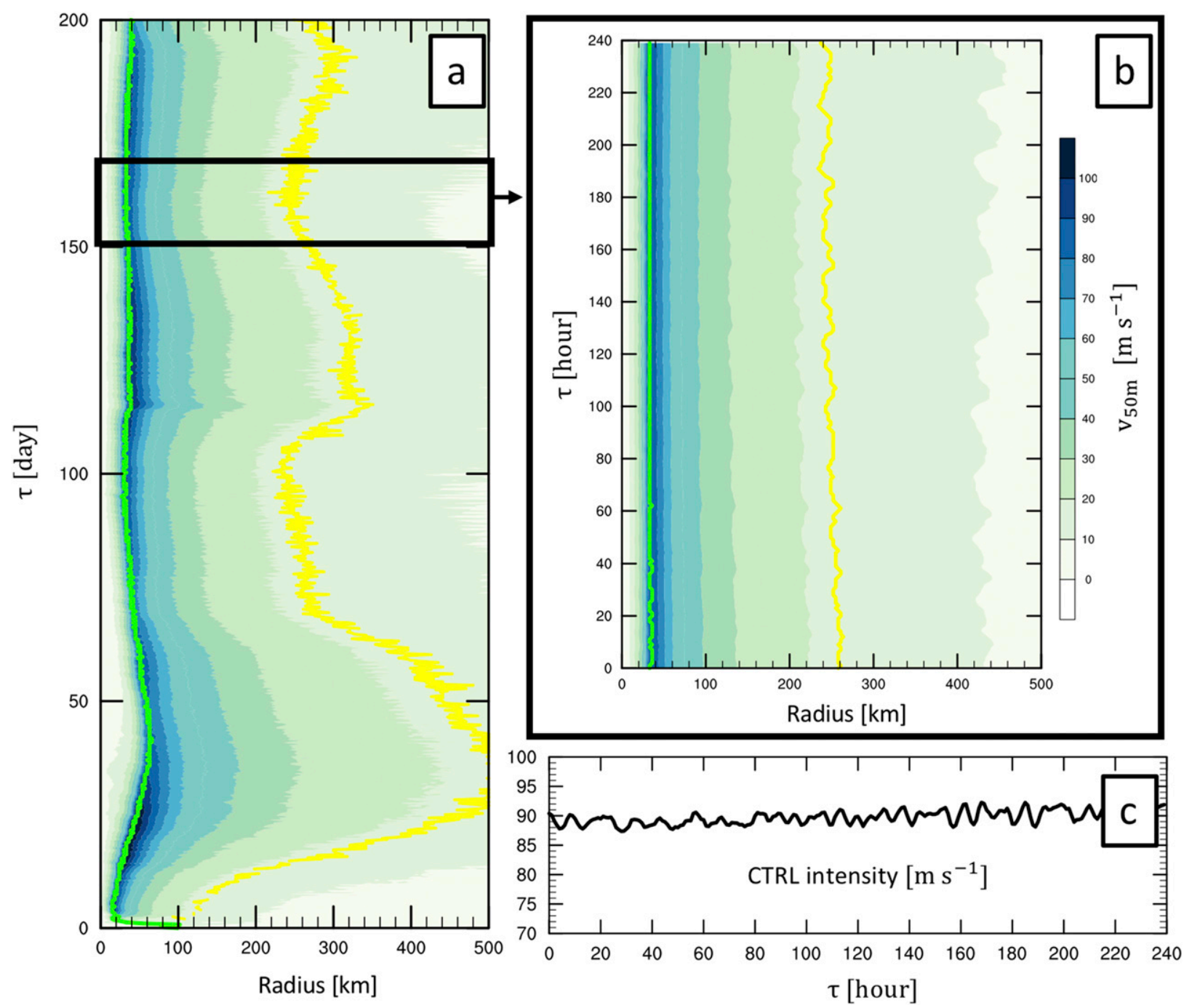

FIG. 1. Hovmöller diagrams of the tangential wind field at the lowest model level (50 m) for (a) the baseline 200-day ocean-surface simulation and (b) the ensemble-mean 10-day CTRL experiment generated from days 150-165 of the baseline simulation, and (c) the evolution of intensity $v_{m}$ for CTRL. Contours in the Hovmöller diagrams denote $r_{\text {max }}$ (green) and $r_{34 \mathrm{kt}}$ (yellow).

in nature and simulations (Persing and Montgomery 2003; Yang et al. 2007; Bryan and Rotunno 2009a,b; Rousseau-Rizzi and Emanuel 2019). Moreover, the model's radial mixing length has a strong influence on the actual $v_{m}$ in axisymmetric simulations (Bryan and Rotunno 2009a; Chavas and Emanuel 2014). We do not address those complex issues here; instead, our focus is on the responses relative to CTRL. Notably, this normalized response approach conveniently yields very similar results when calculating $V_{p}$ from environmental data versus at the radius of maximum wind (Table $\mathrm{S} 1$ in the online supplemental material), with slightly larger disagreement for $0.1 \varepsilon$ experiment where sensible heat fluxes become increasingly important (appendix and
Figs. A1a,b). In our analyses below, we use $V_{p}$ calculated from the environment, which may be considered as a true environmental parameter.

We then seek to analyze the responses of the radial structure of the low-level $(50 \mathrm{~m})$ tangential wind field and precipitation field in greater depth using a representative experiment for each experiment type: $0.25 \varepsilon$ and $4 C_{d}$. These two experiments yield a similar predicted potential intensity response of approximately 0.5 [Eq. (12)]. Moreover, experimentally, they produce strong responses while also retaining a fully coherent vortex throughout the experiment (section 3). These two representative experiments are further used to understand the physical mechanisms underlying the responses. 
To understand the wind field responses, we perform budget analysis for the response in the absolute angular momentum (per unit mass) $M$, given by

$$
M=v r+0.5 f r^{2}
$$

where $v$ is the tangential wind, $r$ is radius, and $f$ is the Coriolis parameter; $M$ is widely used for tropical cyclones since the radial structure of the tangential wind field is directly linked to the radial distribution of $M$ via Eq. (13). Moreover, $M$ is theoretically convenient, because it increases monotonically with radius and is nearly conserved away from frictional boundaries (Emanuel 1986; Chavas et al. 2015; Emanuel and Rotunno 2011; Peng et al. 2018). Note that $M$ may temporarily decrease locally with radius (Smith et al. 2018), although such a state is inertially unstable.

We define the absolute angular momentum response $\Delta M$ as the difference between experiment and CTRL. To understand key processes governing the evolution of this response, we then define a budget equation for $\Delta M$. This begins from the traditional angular momentum budget, which is commonly used in past studies to understand the dynamics of storm circulation (Tuleya and Kurihara 1975). In CM1, the budget equation for $M$ is formulated from the $v$-tendency equation as

$$
\frac{\partial M}{\partial t}=-u \frac{\partial M}{\partial r}-w \frac{\partial M}{\partial z}+r T_{v}+r D_{v}
$$

The first two terms on the rhs of Eq. (14) are the $M$ tendencies associated with radial and vertical advection; $r T_{v}$ is the tendency of $M$ due to turbulent stresses, including surface drag, and $r D_{v}$ is the tendency of $M$ due to diffusion ( $T_{v}$ and $D_{v}$ are applied directly to the $v$-tendency equation within CM1). Within the boundary layer, the direct loss of $M$ due to frictional dissipation is represented by negative values of $r T_{v}$. As noted above, subgrid stress terms on the model bottom boundary are modulated by $C_{d}$ via the frictional velocity $u_{*}$. Increasing $C_{d}$ enhances the surface sink of $M$ (for cyclonic flow) via the $r T_{v}$ term since $T_{v}$ is a function of the subgrid stress. Here the first two terms of Eq. (14) are calculated from model output of $v$, while the latter two are calculated from model output for $T_{v}$ and $D_{v}$. Budget analysis indicates that the diffusion tendency term $r D_{v}$ is not important and so will not be discussed further in this study. From Eq. (14), we may then define a budget equation for $\Delta M$, given by

$$
\frac{\partial(\Delta M)}{\partial t}=\Delta\left(\frac{\partial M}{\partial t}\right)=\Delta\left(-u \frac{\partial M}{\partial r}\right)+\Delta\left(-w \frac{\partial M}{\partial z}\right)+\Delta\left(r T_{v}\right) .
$$

Equation (15) can be applied directly to compare and contrast the underlying processes governing the angular momentum responses in each representative simulation. To minimize transient noise unrelated to the dynamics of spin down, for snapshot analyses we average our minute-by-minute model outputs every hour (1-h average centered on given time). To understand the precipitation responses, we decompose the precipitation response into dynamic and thermodynamic contributions following a simplified decomposition, which is detailed in section $5 b$.

To understand both $M$ and precipitation responses, we also quantify the dynamical responses of the secondary circulation and the thermodynamic responses in surface water vapor mixing ratio fluxes and equivalent potential temperature. The secondary circulation is commonly quantified using the mass streamfunction $\psi$ (e.g., Willoughby 1979). In axisymmetric geometry, the streamfunction is defined as

$$
\frac{\partial \psi}{\partial z}=-r \rho u, \quad \frac{\partial \psi}{\partial r}=r \rho w
$$

$\psi$ can be approximated by integrating the first equation vertically upward or the second equation radially outwards, e.g.,

$$
\psi=\int_{0}^{r} r \rho w d r
$$

where $\psi=0$ at inner boundary $r=0 \mathrm{~km}$. To reduce the complexity of integrating a stretched vertical grid (Zhang et al. 2016), here we integrate the radial equation to calculate $\psi(r, z)$ from model output as

$$
\psi\left(r_{i+\frac{1}{2}}, z\right)=\sum_{i=1}^{N} r_{i, z} \rho_{i, z} w_{i, z} \delta r
$$

where $\rho_{i, z}, w_{i, z}$, and $r_{i, z}$ are the density, vertical velocity and radius of the $i$ th radial grid box; $\delta r=3 \mathrm{~km}$ is the radial grid resolution; and $i=1$ corresponds to $\delta r / 2=1.5 \mathrm{~km}$. We define the secondary circulation response, $\Delta \psi$, as the difference in mass streamfunction between experiments and CTRL. Note that $\psi$ is most accurately calculated by solving a Poisson equation for the entire flow field, though this is much more complex particularly given our vertically stretched grid. Our simpler approach is common in practice (Cook 2004; Liu and Alexander 2007), including in the NCAR Command Language (NCAR 2019).

\section{Temporal evolution of the transient responses to surface roughening and drying}

We first analyze the transient response of storm intensity $v_{m}$ relative to the CTRL (Figs. 2a,b), as well as 

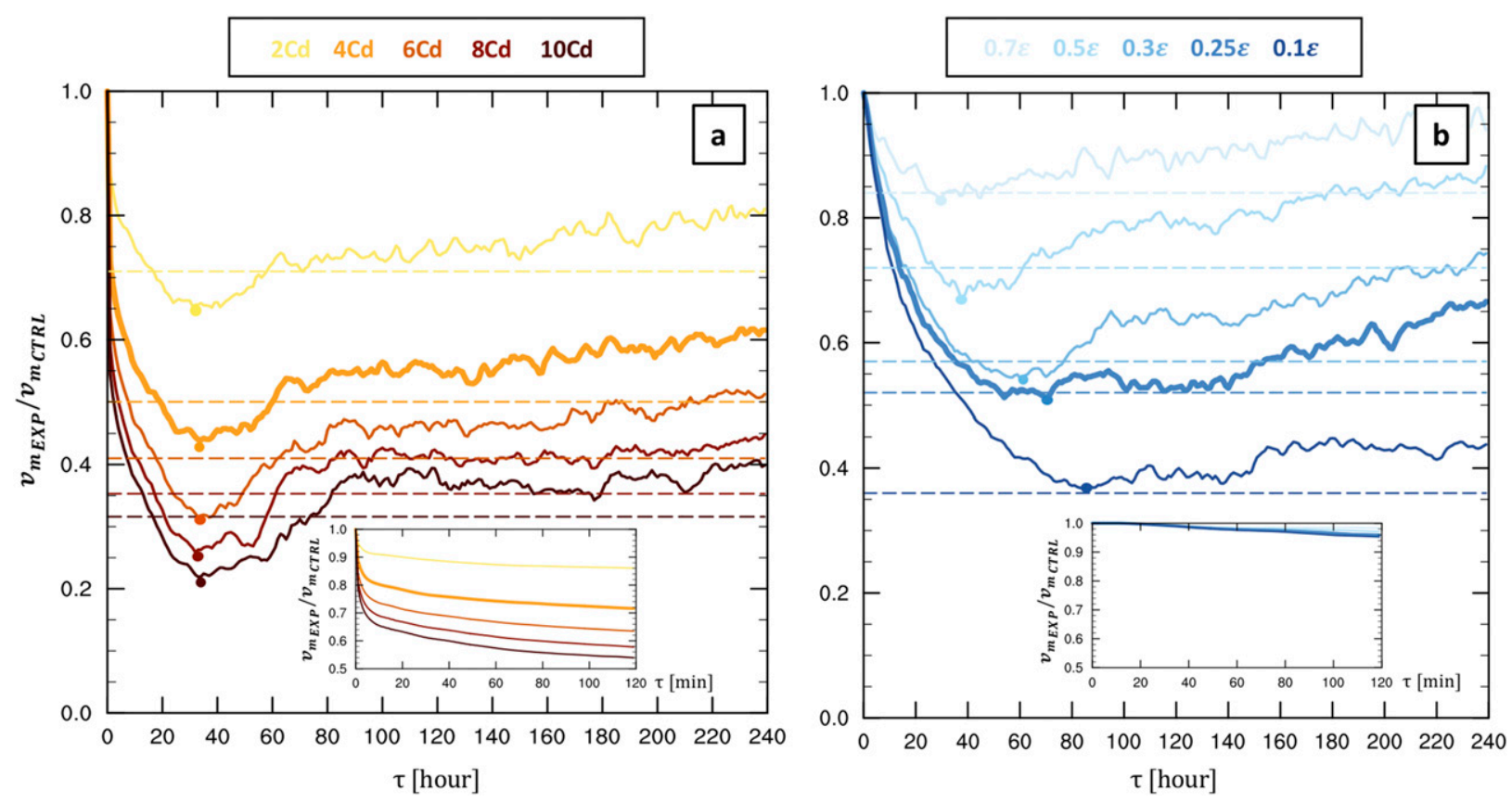

FIG. 2. Temporal evolution of normalized storm intensity $v_{m_{\mathrm{EXP}}} / v_{m_{\mathrm{CTRL}}}$ for (a) surface roughening and (b) surface drying, with the theoretical prediction (dashed line) and minimum normalized intensity (filled circle) for each experiment. Subplots show the first 120 min of change for each experiment. Dashed theoretical predictions are calculated from environmental $V_{p}$ [Eq. (12)]. Thick solid lines correspond to the $4 C_{d}$ and $0.25 \varepsilon$ experiments, which are analyzed in depth. The initial CTRL $v_{m}$ is $90.4 \mathrm{~m} \mathrm{~s}^{-1} ; v_{m}$ is normalized using the time-varying values from CTRL.

the theoretical response given by Eq. (12). Storm intensity decreases monotonically with increasing roughening or drying across all experiments. However, the transient responses to roughening versus drying differ markedly in their intrinsic response time scales.

For surface roughening, $v_{m}$ initially exhibits a very rapid decay across all experiments (Fig. 2a). Notably, there is not a transient period of intensification with roughening that has been found in previous simulations of frictional vortex spin down (Montgomery et al. 2001). The $v_{m}$ then decays more slowly toward a minimum intensity that is smaller than the theoretical response by $\tau=$ $40 \mathrm{~h}$. Thereafter, $v_{m}$ reintensifies to a new equilibrium that is slightly larger than the theoretical response by $\tau=80 \mathrm{~h}$, and this equilibrium lasts at least 2 days. The time scales of this transient response in $v_{m}$ appear to be independent of the roughening magnitude; the roughening magnitude primarily modulates the magnitude of the response.

In contrast, for surface drying, $v_{m}$ responds negligibly within the first hour and only weakly by $\tau=2 \mathrm{~h}$ (subplot in Fig. 2b). The $v_{m}$ then decays slowly and quasi exponentially toward its minimum value that is comparable to the theoretical equilibrium response; $v_{m}$ retains this minimum value for approximately 2 days for $0.1 \varepsilon$ and $0.25 \varepsilon$ and 1 day for $0.3 \varepsilon, 0.5 \varepsilon$ and $0.7 \varepsilon$, with very gradual reintensification thereafter. The existence of this new equilibrium state follows from potential intensity theory, which predicts that the storm should tend toward a new, weaker but nonzero equilibrium, as surface enthalpy fluxes are reduced but remain nonzero (Kieu 2015; Chavas 2017), including both latent and sensible heat fluxes. Deeper analysis of long-term behavior at and beyond this equilibrium state is left to future work. The time scale of the transient response monotonically increases with increased magnitude drying, again in contrast to surface roughening. For both roughening and drying, the minimum surface pressure $P_{\mathrm{MIN}}$ increases by $\tau=40 \mathrm{~h}$ (Fig. S1 in the online supplemental material), exhibiting similar transient time scales as $v_{m}$. Future work might seek to analyze potential changes in the wind-pressure relationship due to surface forcing (e.g., Kieu and Zhang 2010).

The nature of the responses in $r_{\text {max }}$ and $\mathrm{r}_{34 \mathrm{kt}}$ (Figs. 3a,b) are similar to that of $v_{m}$ for roughening: an initial rapid decrease followed by a more gradual decrease toward a new smaller equilibrium. $r_{\max }$ exhibits slightly more variation within the first $18 \mathrm{~h}$, decreasing sharply within $\tau=1 \mathrm{~h}$, then becoming constant through $\tau=12 \mathrm{~h}$, and then briefly increasing by $\tau=18 \mathrm{~h}$. Thereafter, $r_{\max }$ decays rapidly again at a rate that increases with increased roughness. After $\tau=60 \mathrm{~h}, r_{\max }$ becomes relatively constant. 

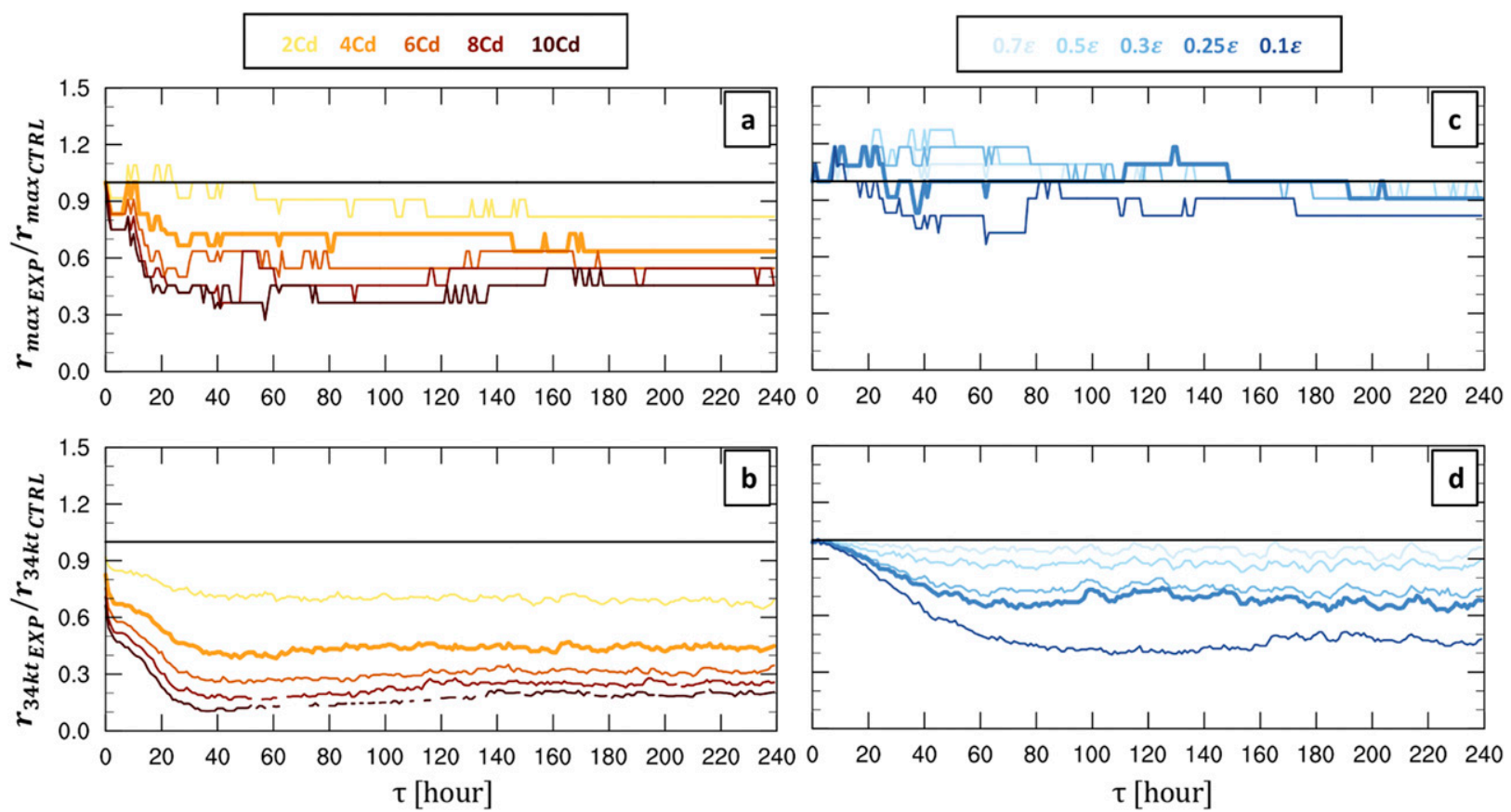

FIG. 3. Temporal evolution of normalized storm size (top) $r_{\max _{\mathrm{EXP}}} / r_{\max _{\mathrm{CTRL}}}$ and (bottom) $r_{34 \mathrm{kt} \mathrm{EXP}_{\mathrm{XP}}} / r_{34 \mathrm{kt} \text { CTRL }}$ for (a), (b) surface roughening and (c),(d) surface drying. The initial CTRL $r_{\max }$ and $r_{34 \mathrm{kt}}$ are 33 and $258 \mathrm{~km}$, respectively. The aesthetics are as in Fig. 2.

For drying, $r_{34 \mathrm{kt}}$ decreases slowly toward its minimum and then becomes stable (Figs. 3c,d), which is similar to its $v_{m}$ evolution. Meanwhile, the response of $r_{\max }$ is more complex and nonmonotonic. Initially, $r_{\max }$ remains relatively constant or slightly increases across all drying experiments up to $20 \mathrm{~h}$. Thereafter, though, $r_{\max }$ decreases with a magnitude that increases with increasing drying, eventually returning to a radius close to the CTRL $r_{\max }$. For example, for strong surface drying $(0.1 \varepsilon), r_{\max }$ decreases to $70 \%$ of its CTRL value by $\tau=$ $40 \mathrm{~h}$, then returns to around $85 \%$ of the CTRL value. In contrast, $r_{\max }$ remains larger than CTRL for weaker drying; for $0.25 \varepsilon, r_{\max }$ gradually decreases back toward the CTRL value by $40 \mathrm{~h}$ and fluctuates around this value thereafter.

We also analyze the total precipitation response within $r<150 \mathrm{~km}$. With roughening (Fig. 4a), $P$ initially increases by $15 \%-40 \%$ from $\tau=0$ to $5 \mathrm{~h}$, with larger enhancement and peak value for stronger magnitude roughening. This transient precipitation enhancement consistently peaks at $\tau=5 \mathrm{~h}$, independent of roughening magnitude. $P$ subsequently decreases by $20 \%-80 \%$ of the CTRL value from $\tau=5$ to $20 \mathrm{~h}$, with larger precipitation reduction for stronger roughening. After remaining relatively constant for approximately 1 day, $P$ gradually increases back toward the CTRL value and reequilibrates after $\tau=100 \mathrm{~h}$ across all roughening experiments. Meanwhile, for surface drying experiments,
$P$ decreases by $30 \%-90 \%$ before reaching a new equilibrium, with larger reduction for larger magnitude drying (Fig. 4b). The intrinsic time scales of this response remain constant for roughening while increase with increased drying, similar to the results above for $v_{m}$ and $r_{34 \mathrm{kt}}$.

These two sets of experiments demonstrate the systematic transient responses of TC intensity, size, and inner-core precipitation rate to surface roughening and drying across a range of magnitudes of each parameter. For roughening, the intrinsic time scales of these responses are independent of roughening magnitude. In contrast, for drying, the responses are generally smoother and follow a single dominant time scale that varies with drying magnitude, with the exception of $r_{\max }$ whose qualitative response exhibits a more complex dependence on the strength of drying.

\section{Radial structure of the response in low-level wind field and precipitation field}

We next perform an in-depth analysis of one representative experiment from each set: $0.25 \varepsilon$ and $4 C_{d}$ (bold lines in Figs. 2-4). We focus on the first $40 \mathrm{~h}$, during which intensity decreases rapidly and monotonically across all roughening and drying experiments. The slow longer-term reintensification, which is likely tied to the gradual adjustment of the environment to these changes 

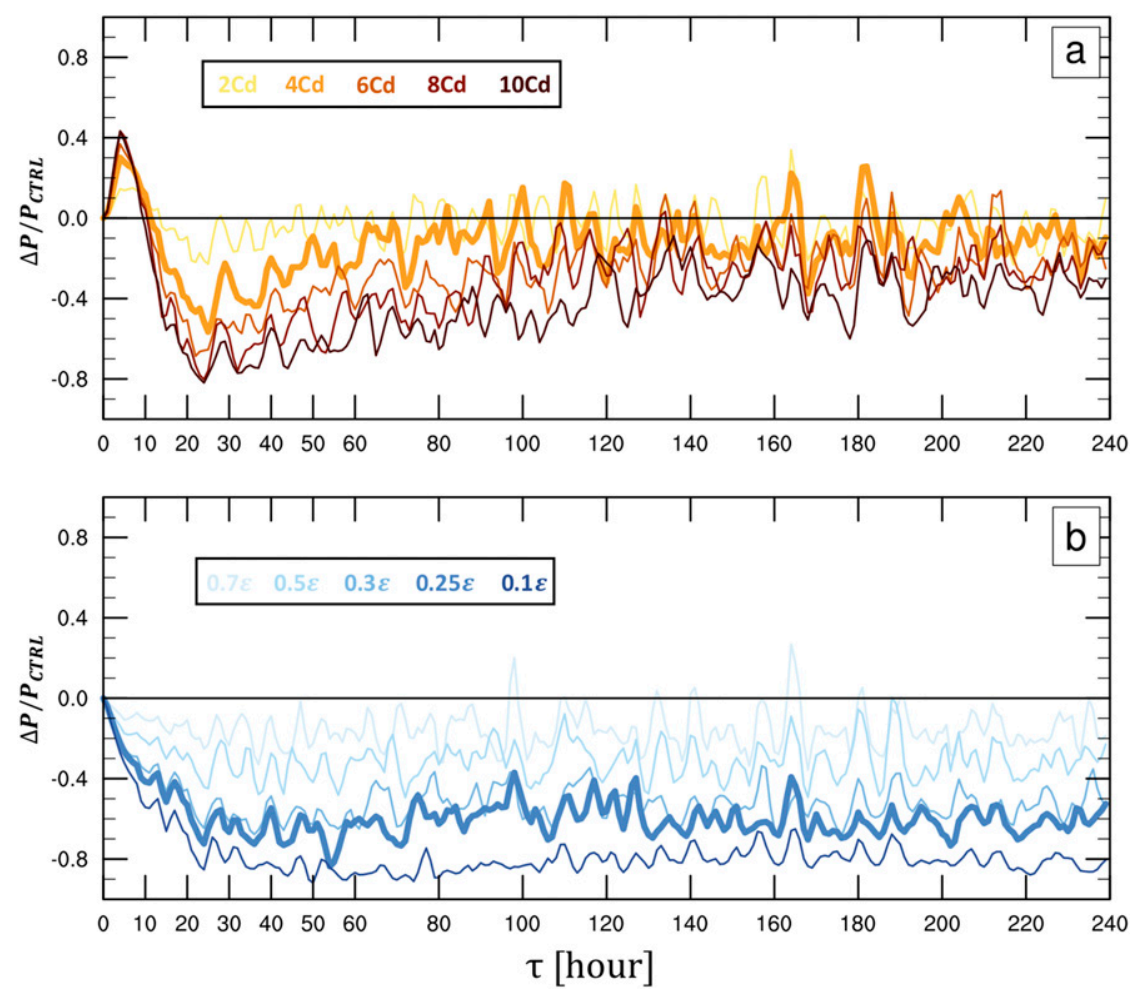

FIG. 4. Temporal evolution of normalized response in total precipitation, $\Delta P / P_{\mathrm{CTRL}}$, for (a) surface roughening and (b) surface drying. The initial CTRL $P$ is $763.3 \mathrm{~mm} \mathrm{~h}^{-1}$. The aesthetics are as in Fig. 2.

in surface properties, is generally less relevant to our primary research questions with regard to landfall and so is left for future work.

How do changes in TC intensity and size manifest themselves in the response of the radial structure of the low-level wind field? Though the nature of this weakening differs markedly between drying and roughening, the near-surface and low-level tangential wind field outside of the eye weakens monotonically during the first two days (Fig. 5); within the eye, wind speeds increase after 1-2 days. Surface drying predominantly weakens the inner-core wind field slowly by $\tau=12 \mathrm{~h}$, whereas the response of the broad outer circulation is weaker moving outwards to larger radii, with limited
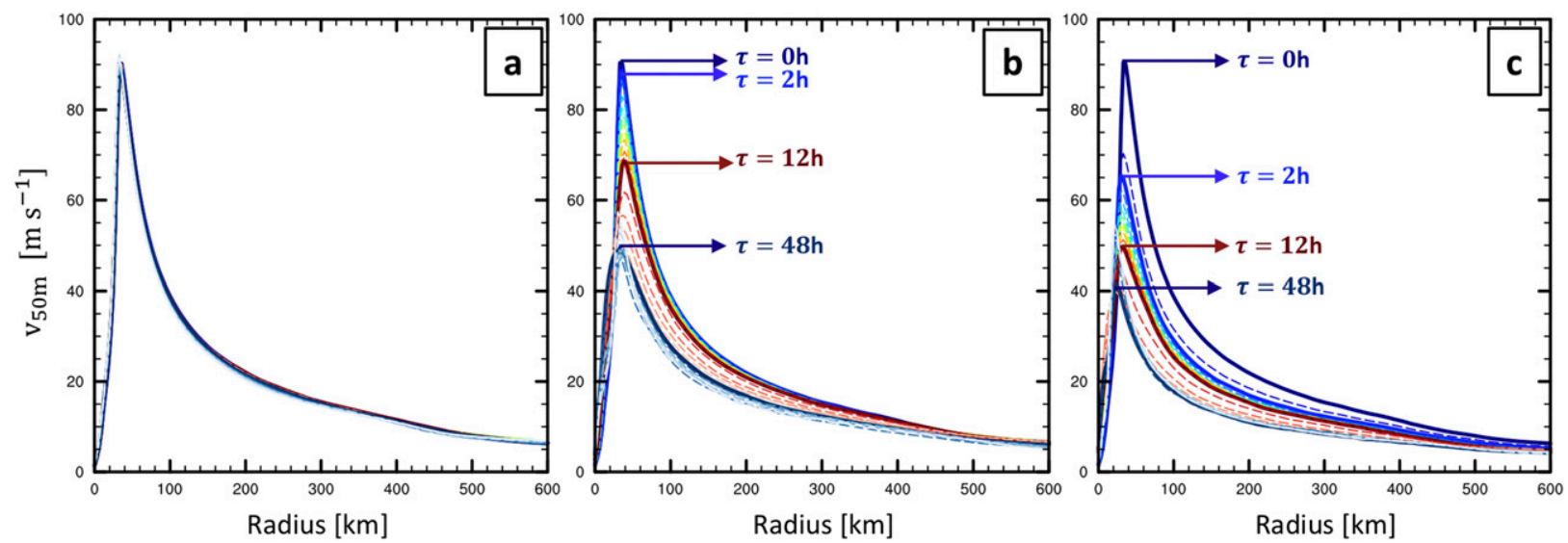

FIG. 5. Ten-day evolution of the 50-m tangential wind profile for (a) CTRL, (b) $0.25 \varepsilon$, and (c) $4 C_{d}$. Wind profiles are shown every hour for $\tau=0-12 \mathrm{~h}$ (rainbow colors), every $6 \mathrm{~h}$ from $\tau=12$ to $48 \mathrm{~h}$ (from dark to light red), and every $24 \mathrm{~h}$ from $\tau=48$ to $240 \mathrm{~h}$ (from dark to light blue). Wind fields at $\tau=0,2,12$, and $48 \mathrm{~h}$ are marked and are plotted in thick solid lines in sequence. 

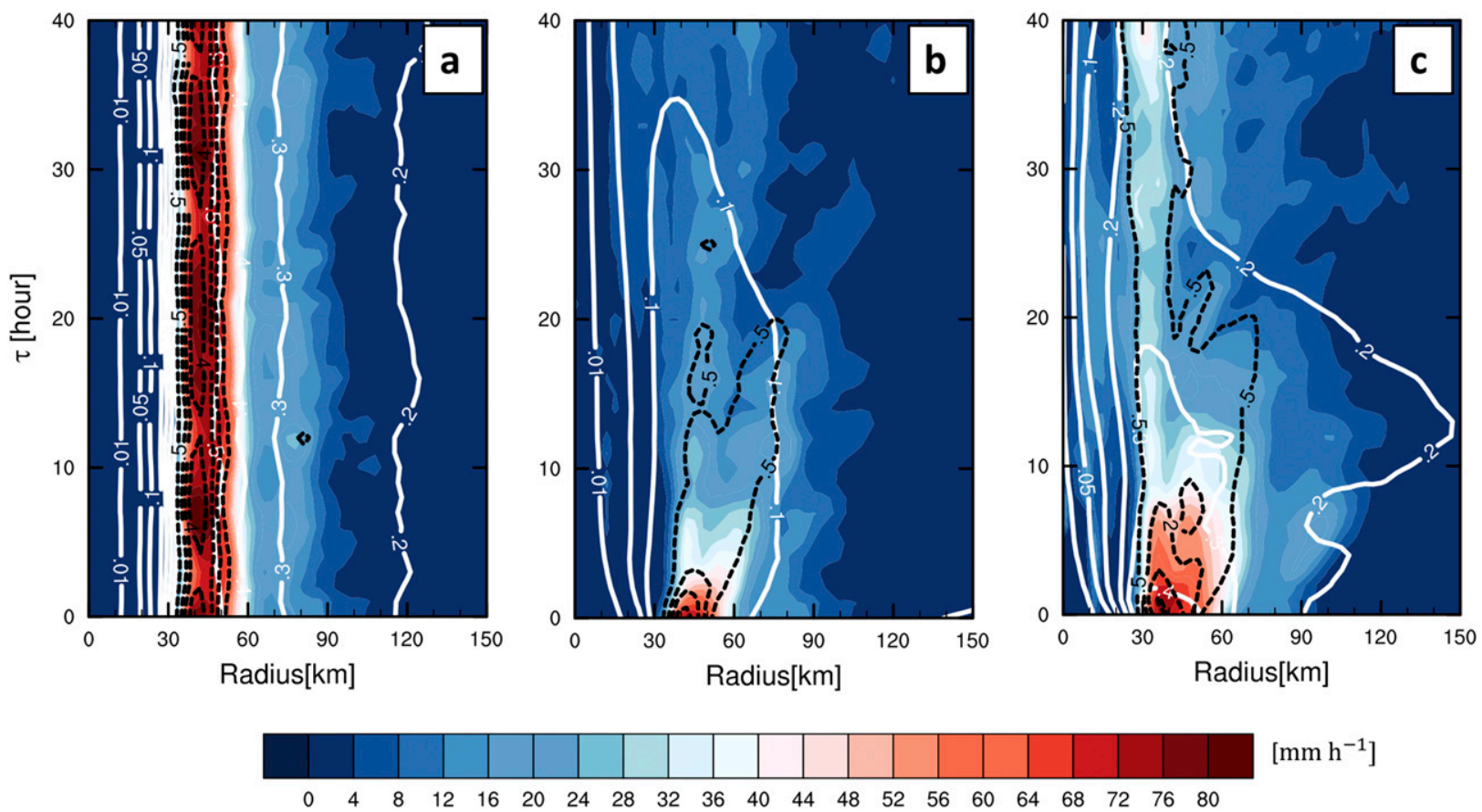

FIG. 6. Hovmöller diagram of precipitation (colors), hourly averaged $w_{2 \mathrm{~km}}\left(\mathrm{~m} \mathrm{~s}^{-1}\right.$; dashed contours), and $F_{\mathrm{qv}}\left(\mathrm{g} \mathrm{kg}^{-1} \mathrm{~m} \mathrm{~s}^{-1}\right.$; solid contours) for (a) CTRL, (b) $0.25 \varepsilon$, and (c) $4 C_{d}$ from $\tau=0$ to $40 \mathrm{~h}$.

weakening beyond $r=100 \mathrm{~km}$ by $\tau=12 \mathrm{~h}$ and modest weakening at $r=500 \mathrm{~km}$ by $\tau=48 \mathrm{~h}$ (Fig. $5 \mathrm{~b}$ ). In contrast, surface roughening weakens the low-level wind field at all radii strongly and rapidly within the first two hours and more slowly thereafter (Fig. 5c). Responses of the outer circulation between drying and roughening experiments also explain the evolution of $r_{34 \mathrm{kt}}$ (Figs. 3d,b): for surface drying, $r_{34 \mathrm{kt}}$ changes modestly over the first $24 \mathrm{~h}$ due to the negligible initial response of the TC outer circulation. In contrast, when the complete low-level wind field weakens rapidly due to surface roughening, wind speeds at the original $r_{34 \mathrm{kt}}(r=258 \mathrm{~km})$ also decrease well below $34 \mathrm{kt}\left(17.5 \mathrm{~m} \mathrm{~s}^{-1}\right)$ rapidly. Thus, $r_{34 \mathrm{kt}}$ shifts inward to a much smaller radius.

As noted in section 3, total inner-core precipitation responds differently to surface drying and roughening: by $\tau=5 \mathrm{~h}, P$ is $25 \%$ lower for $0.25 \varepsilon$ and $30 \%$ higher than CTRL for $4 C_{d}$ (Fig. 4). Here we further compare the spatiotemporal distribution of rainfall, in conjunction with surface mixing ratio fluxes $F_{\mathrm{qv}}$ and $2-\mathrm{km}$ updraft $w_{2 \mathrm{~km}}$ (Fig. 6); the latter two variables will be discussed in the mechanistic analysis below. For surface drying, heavy precipitation exceeding $50 \mathrm{~mm} \mathrm{~h}^{-1}$ quickly diminishes to below $30 \mathrm{~mm} \mathrm{~h}^{-1}$ after $\tau=5 \mathrm{~h}$ (Fig. 6b). By $\tau=10 \mathrm{~h}$, precipitation primarily decreases within the inner core, leaving a relatively constant radial extent of lighter precipitation rates less than $20 \mathrm{~mm} \mathrm{~h}^{-1}$, which is consistent with the response of the low-level wind field.
For surface roughening, heavy precipitation expands both inwards and outwards from $r=40-55 \mathrm{~km}$ in CTRL to $r=30-60 \mathrm{~km}$ during $\tau<5 \mathrm{~h}$ (Figs. 6a,c). Meanwhile, the region of lighter precipitation rates expands to larger radii beyond $r=90 \mathrm{~km}$. After $\tau=5 \mathrm{~h}$, the region of heavier precipitation disappears, yet lighter precipitation persists beyond $r=90 \mathrm{~km}$. After $\tau=10 \mathrm{~h}$, a concentrated annulus of higher precipitation gradually reemerges associated with the redevelopment of the eyewall at $r=30 \mathrm{~km}$. Deeper analysis of precipitation changes is provided in section $5 \mathrm{~b}$.

\section{Physical mechanisms}

We next seek a detailed mechanistic understanding of the responses to roughening and drying characterized above. We again focus on our two representative experiments: $0.25 \varepsilon$ and $4 C_{d}$. Since the responses to surface drying follow a single dominant time scale as described in sections 3 and 4 , we begin our analysis with the $0.25 \varepsilon$ experiment.

\section{a. Low-level wind field}

To understand the responses of the low-level wind field, we quantify the responses of absolute angular momentum, $\Delta M$ (Fig. 7), and analyze the physical processes controlling $\Delta M$ for $0.25 \varepsilon$ (Fig. 8) and $4 C_{d}$ (Fig. 9). We plot pointwise differences from CTRL rather than 


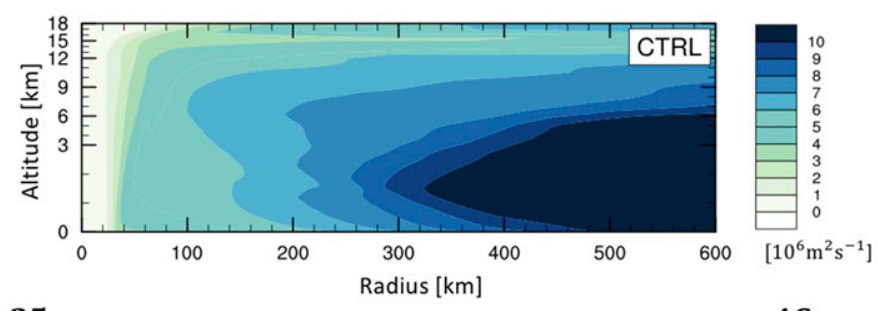

$0.25 \varepsilon$
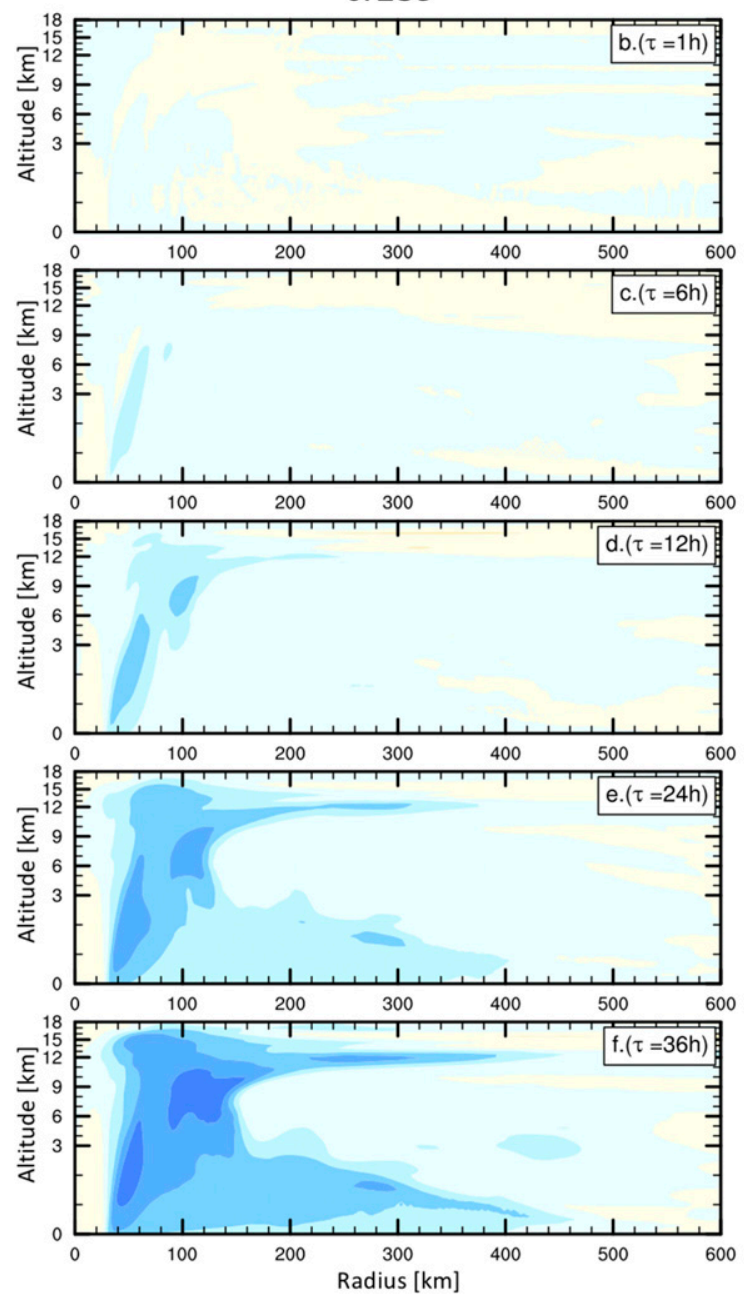
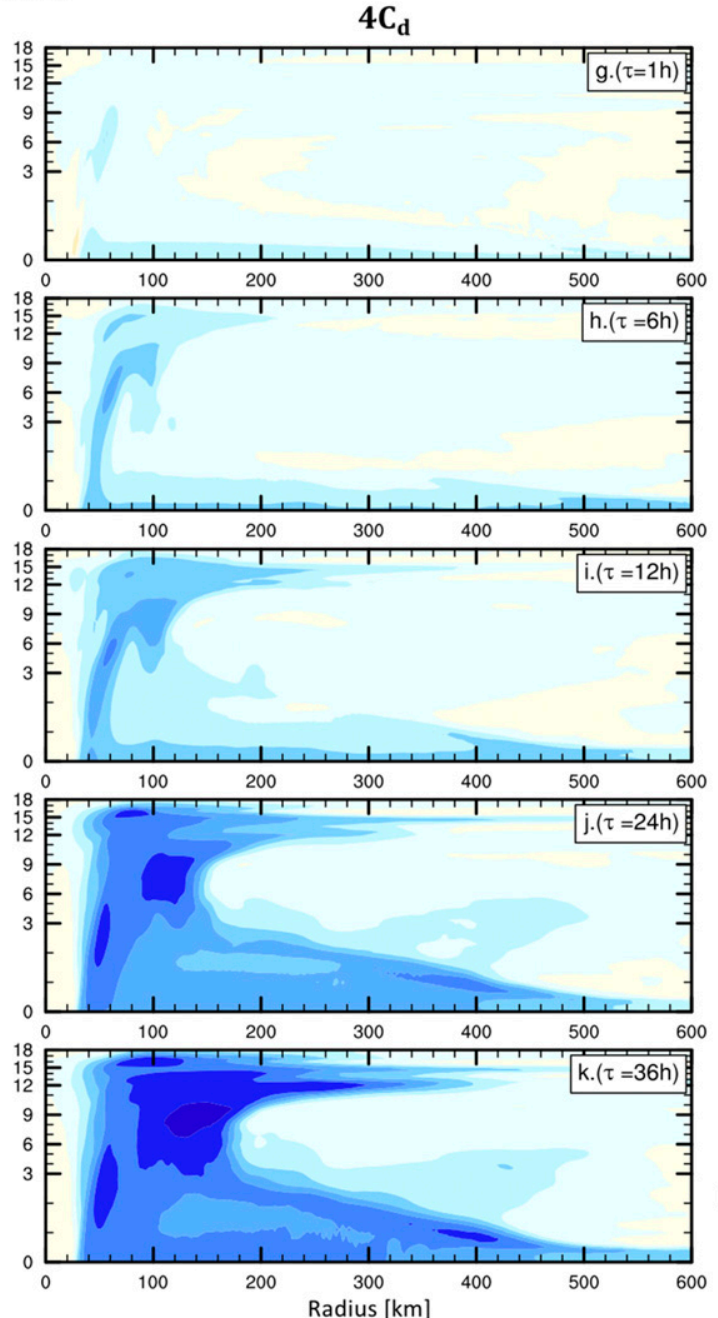
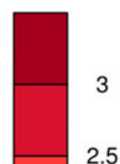

2

1.5

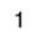

0.5

0

FIG. 7. (a) CTRL angular momentum field, and responses of angular momentum $\Delta M$ at $\tau=$ (b),(g) 1 , (c),(h) 6, (d),(i) 12 , (e),(j) 24, and (f),(k) $36 \mathrm{~h}$ for (left) $0.25 \varepsilon$, and (right) $4 C_{d}$. Values are calculated as 1-h averages centered on a given time. CTRL shows the 1-h average centered at $\tau=1 \mathrm{~h}$.

side-by-side comparisons to highlight the detailed structure of the responses.

\section{1) RESPONSE TO SURFACE DRYING $(0.25 \varepsilon)$}

For surface drying, $M$ changes minimally throughout the whole domain during the first hour (Fig. 7b). By $\tau=$ $6 \mathrm{~h}, M$ begins to decrease within the eyewall aloft, while the near-surface $M$ remains comparable to the CTRL (Fig. 7c). By $\tau=12 \mathrm{~h}$, this response extends upward into the outflow, whereas within the boundary layer it remains confined to near the radius of maximum wind (Fig. 7d). During $\tau=12-24 \mathrm{~h}$, the response extends radially outward at low levels (Fig. 7e). By $\tau=36 \mathrm{~h}$, the response pattern is similar to $24 \mathrm{~h}$ but with stronger magnitude: $(\Delta M)_{\max }$ reaches $-1.5 \times 10^{6} \mathrm{~m}^{2} \mathrm{~s}^{-1}$ near the outflow region (Fig. 7f). As a result, the low-level wind field weakens slowly and primarily within the inner region during the first $2 \mathrm{~h}$, as found in section 4 . 

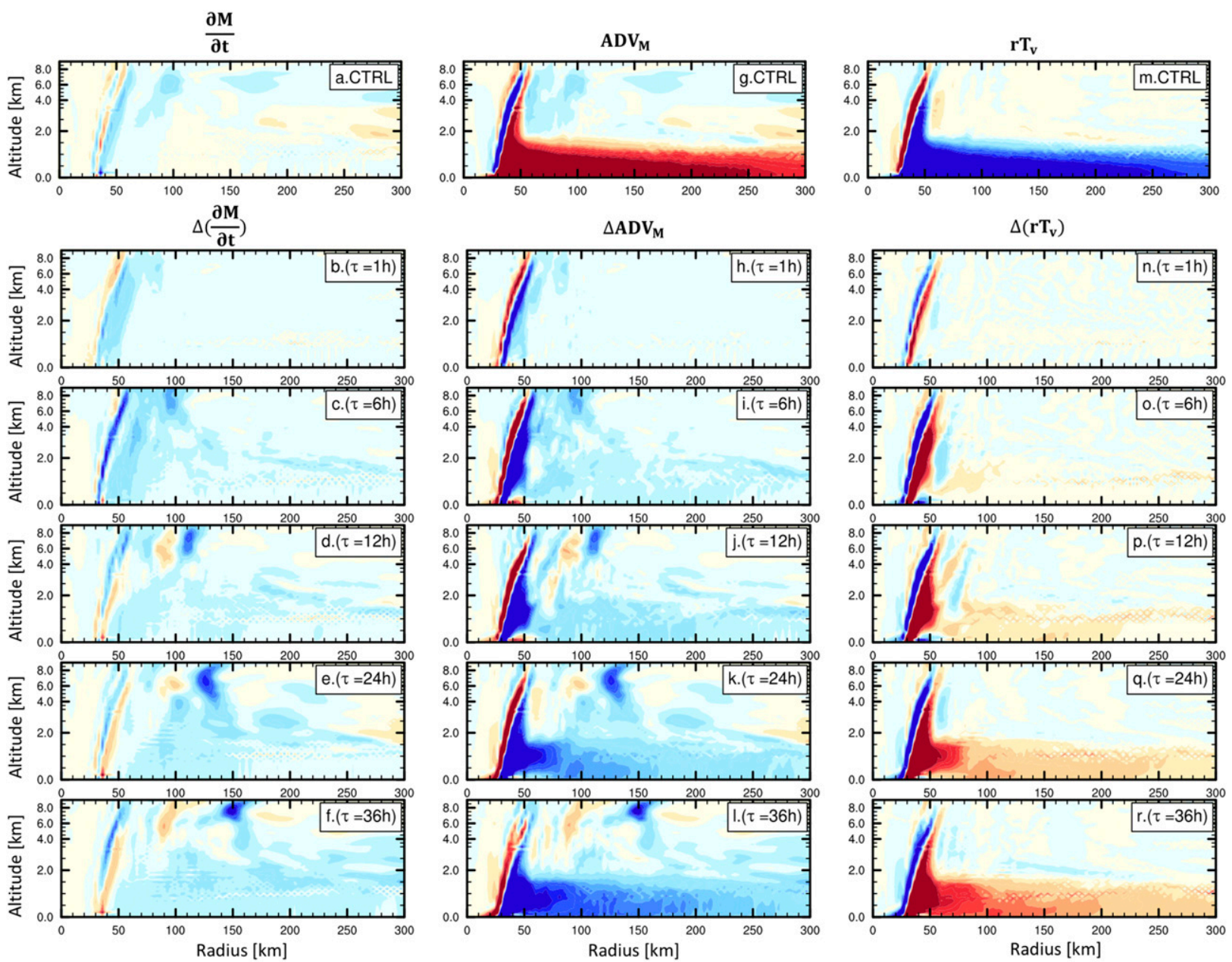

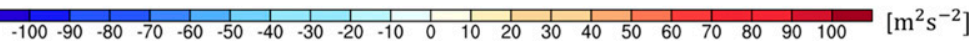

FIG. 8. Angular momentum budget for CTRL and evolution of the response angular momentum budget for $0.25 \varepsilon$. Terms include (a)-(f) angular momentum tendency, $(\mathrm{g})-(\mathrm{l})$ advection, and $(\mathrm{m})-(\mathrm{r})$ turbulent mixing. Values are calculated as 1-h averages centered on a given time. CTRL shows the 1 -h average centered at $\tau=1 \mathrm{~h}$.

We use Eq. (15) to quantify how changes in advection and turbulent mixing, including surface frictional dissipation, control the response tendency, $\Delta(\partial M / \partial t)$, and thus $\Delta M$ (Fig. 8). We first combine $\Delta[-u(\partial M / \partial r)]$ and $\Delta[-w(\partial M / \partial z)]$ into a total advection response term, $\triangle \mathrm{ADV}_{M}$. Within the inner-region boundary layer, the dominant source term of $M$ is due to advection (Fig. $8 \mathrm{~g}$ ) and the dominant sink term is due to turbulent dissipation by surface friction (Fig. 8m); the latter depends on both surface drag coefficient and near-surface total wind speed [Eqs. (9)-(11)]. The evolution of $\Delta(\partial M / \partial t)$ is controlled primarily by the decreasing advective $M$ source near the surface (Figs. $8 \mathrm{~h}-1$ ). Changes in $M$ loss by turbulent dissipation, $\Delta\left(r T_{v}\right)$, depends solely on changes in near-surface wind speed since $C_{d}$ is fixed. Thus, for $\tau=0-2 \mathrm{~h}$, during which the wind field remains nearly constant (Fig. 5b), near-surface $\Delta\left(r T_{v}\right)$ changes litthe relative to CTRL (Fig. $8 \mathrm{n}$ ). During this time, $\Delta(\partial M / \partial t$ ) remains near zero initially except for the slight radial expansion near the eyewall (Fig. 8b). Through $\tau=36 \mathrm{~h}$, the turbulent sink term monotonically increases toward smaller negative values (i.e., a weaker sink of $M$ ) due to the slowly weakening primary circulation (Figs. 8n-r). Meanwhile, the advective $M$ source is gradually decreasing as well (Figs. 8h-1). However, the advective source is consistently slightly smaller in magnitude than the frictional sink, yielding a monotonically weakening storm (Figs. 8bf). Beyond $\tau=36 \mathrm{~h}$, the weakened frictional $M$ sink and advective $M$ source begin to more closely balance each other as the storm approaches a new, weaker equilibrium.

For deeper analysis of the boundary layer response, we integrate each of the two dominant source/sink terms 

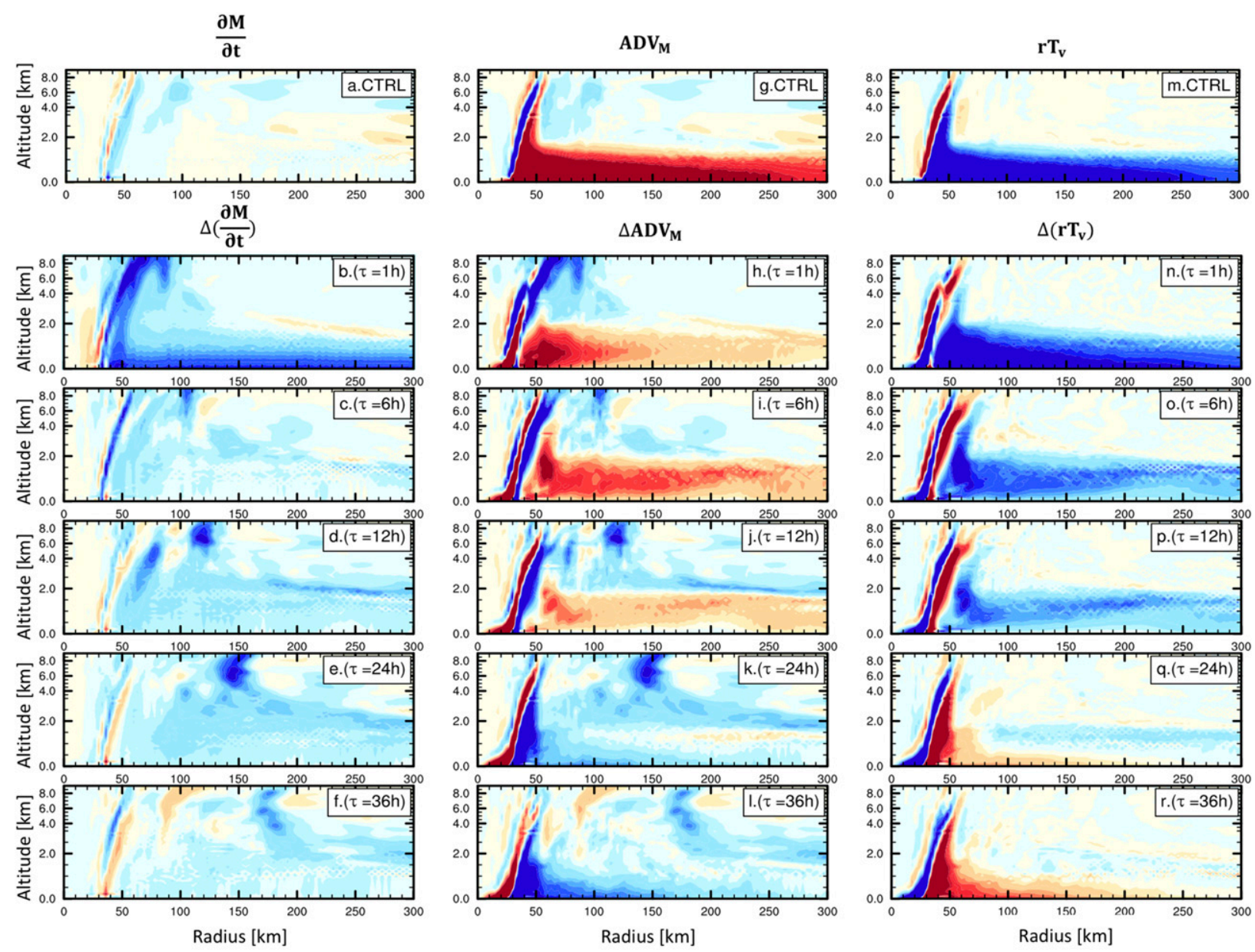

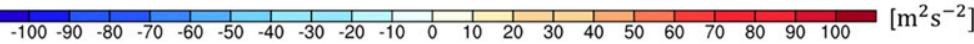

FIG. 9. As in Fig. 8, but for $4 C_{d}$.

within the cylinder bounded by $r \leq 300 \mathrm{~km}$ and $z \leq 2 \mathrm{~km}$; that is,

$$
Y=\int_{0}^{z=2 \mathrm{~km}} \int_{0}^{r=300 \mathrm{~km}} X r \rho d r d z
$$

where $X$ represents the tendency $(\partial M / \partial t)$, the frictional sink $\left(r T_{v}\right)$, or the radial or vertical advection responses $\{[-u(\partial M / \partial r)]$ and $[-w(\partial M / \partial z)]$, respectively\}. The integrated response is then defined as $\Delta Y$. The results for both drying and roughening experiments are shown in Fig. 10. The advection response is generally dominated by the radial component as expected, as this component represents the dominant source of advection for the system within the boundary layer. Comparison among source and sink terms over time [Eq. (19); Fig. 10a] confirms that the inward advective $M$ source is consistently insufficient to balance the frictional dissipation sink, resulting in negative values of $\partial M / \partial t$, that is, the gradual loss of $M$ and thus the weakening of low-level wind field in the inner core with time. We note that this two-region vortex behavior, in which the inner-core intensity changes independent of a relatively stable outer circulation, aligns closely with the physical wind structure model of Chavas and Lin (2016).

\section{2) Response to SURFACE ROUGHENING $\left(4 C_{D}\right)$}

For surface roughening, $M$ initially decreases rapidly near the surface at all radii (Fig. $7 \mathrm{~g}$ ), and this signal subsequently extends upward within the eyewall during the first hour. By $\tau=12 \mathrm{~h}$, the reduction of $M$ extends radially outward within the boundary layer and in the outflow above the eyewall, with $(\Delta M)_{\max }$ exceeding $-2 \times 10^{6} \mathrm{~m}^{2} \mathrm{~s}^{-1}$ (Figs. $7 \mathrm{~h}-\mathrm{i}$ ). During $\tau=12-$ $36 \mathrm{~h}, M$ decays through the depth of the vortex, with largest magnitude changes in the outflow region where $(\Delta M)_{\max }$ exceeds $-3 \times 10^{6} \mathrm{~m}^{2} \mathrm{~s}^{-1}$ (Figs. $7 \mathrm{j}-\mathrm{k}$ ). This reduction of $M$ near the surface at all radii translates 

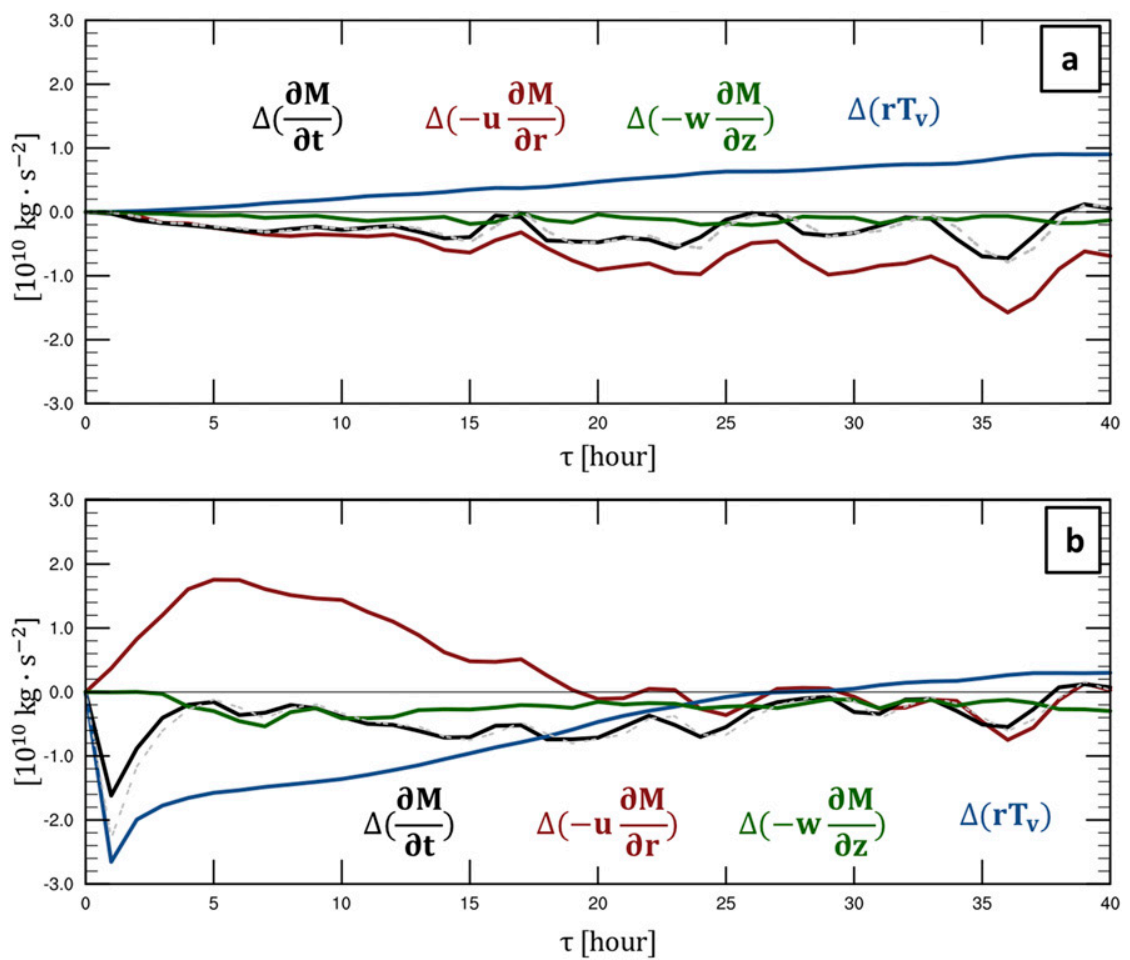

FIG. 10. Temporal evolution of the integrated responses of the angular momentum budget terms [Eq. (19)] for (a) $0.25 \varepsilon$ and (b) $4 C_{d}$. The sums of the three response budget terms (dashed gray lines) are shown to compare with the $\Delta(\partial M / \partial t)$ (black lines).

directly to the strong and rapid weakening of the complete low-level tangential wind field (Fig. 5c).

We again analyze the evolution of $\Delta M$ via budget analysis (Fig. 9). Initially $(\tau<1 \mathrm{~h})$, boundary layer $\partial M / \partial t$ decreases rapidly at all radii (Fig. $9 \mathrm{~b}$ ) due to the greatly enhanced $M$-sink (Fig. 9n), which is forced by the increased drag coefficient $C_{d}$. This enhanced sink exceeds the moderately enhanced advective $M$ source within the inner core $r<100 \mathrm{~km}$ (Fig. 9h). By $\tau=12 \mathrm{~h}$, the storm weakens more slowly, with $\Delta(\partial M / \partial t)$ decreasing in magnitude to $-20 \mathrm{~m}^{2} \mathrm{~s}^{-2}$ (Figs. 9c,d). This decrease results from the reduction in $\Delta\left(r T_{v}\right)$ (Figs. 9o,p) due to the strongly weakened low-level wind speeds (Fig. 5c). Meanwhile, the initial enhancement of $\mathrm{ADV}_{M}$ also decays gradually during this period (Figs. 9i,j), thus coming closer to balancing the frictional $M$-sink. After $\tau=24 \mathrm{~h}, \partial M / \partial t$ gradually decreases toward zero in much of the inner domain (Figs. 9e,f), indicating that the vortex is approaching a new equilibrium. At low levels, the advective source response changes sign and becomes slightly negative (i.e., a weaker source; Figs. 9k,1), while the turbulent sink response becomes slightly positive (i.e., a weaker sink; Figs. 9q,r). This final behavior reflects the much weaker primary and secondary circulations overlying a rougher surface.
We analyze the temporal evolution of the integrated response of $M$ sources and sinks to identify the dominant physical process for the boundary layer $M$ tendency (Fig. 10b). By $\tau=2 \mathrm{~h}$, the frictional sink is enhanced significantly more than the advective source, and this imbalance causes $\Delta(\partial M / \partial t)$ to decrease rapidly. By $\tau=$ $5 \mathrm{~h}$, the advective source response reaches its maximum magnitude, though it is never sufficient to fully balance the surface frictional dissipation source. Thus, $\Delta(\partial M / \partial t) \leq 0$ before reaching a new equilibrium.

It is insightful to compare the evolutions of the integrated responses of the advective $M$ source and frictional $M$ sink terms shown in Fig. 10 across all drying and roughening experiments (Fig. 11). Each integrated response is normalized by its CTRL value, that is, $\Delta Y / Y_{\text {CTRL }}$, where $Y$ represents the integrated radial advective $M$ source or frictional $M$ sink shown in Fig. 10 [Eq. (19)]. All surface drying experiments produce qualitatively similar responses that are amplified with stronger drying, but with time scales that increase with drying magnitudes. All surface roughening experiments also produce qualitatively similar responses that are amplified with stronger roughening, but with constant time scales for the peak enhancement in advective $M$ source by $\tau=5 \mathrm{~h}$ and the reequilibration of both source and sink terms after $\tau=24 \mathrm{~h}$. The distinct time scales of 

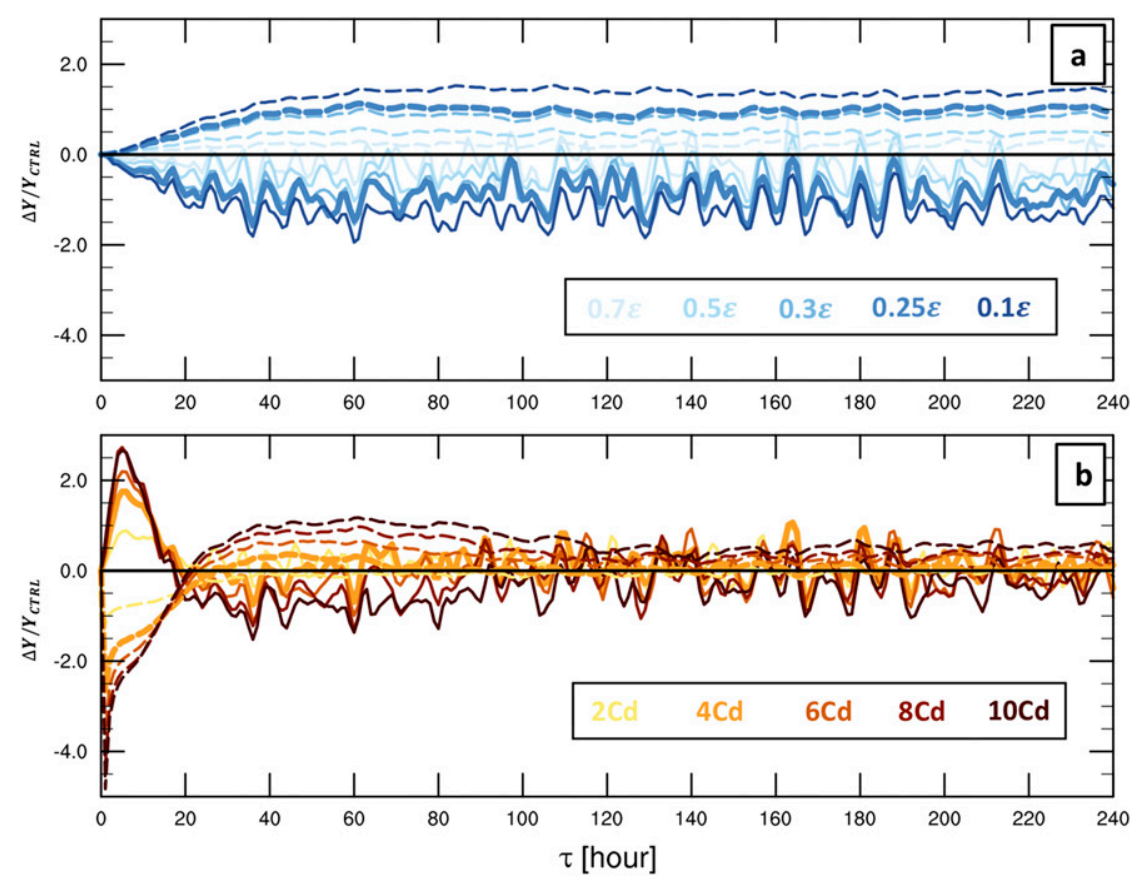

FIG. 11. Temporal evolution of the integrated responses of the radial advective $M$ source \{solid lines; $\Delta[-u(\partial M / \partial r)]$ in Fig. 10\} and turbulent $M$ sink [dashed lines; $\Delta\left(r T_{v}\right)$ in Fig. 10], calculated from Eq. (19) and normalized by the CTRL value of each term, $\Delta Y / Y_{\mathrm{CTRL}}$, for (a) surface drying experiments and (b) surface roughening experiments. The aesthetics are as in Fig. 2.

the storm responses characterized in section 3 appear intimately tied to the responses of these underlying source and sink terms in each experiment.

\section{3) CONTRASTING BOUNDARY LAYER RESPONSES TO ROUGHENING AND DRYING}

Ultimately, the responses of the underlying processes controlling the primary vortex circulation described above (Figs. 8, 9, middle columns) are intimately linked to the responses of the secondary overturning circulation.

To better understand their interdependent dynamics, we first linearly decompose the response advective term to quantify the relative contributions of changes in the primary circulation and secondary circulation during the first $12 \mathrm{~h}$. This decomposition is given by

$$
\begin{aligned}
\Delta \mathrm{ADV}_{M}= & \Delta(\mathbf{U} \cdot \nabla M) \approx \Delta \mathbf{U} \cdot \nabla M+\mathbf{U} \cdot \Delta(\nabla M) \\
& +\Delta \mathbf{U} \cdot \Delta(\nabla M),
\end{aligned}
$$

where $\mathbf{U}=(u, w)$ and $\nabla M=(\partial M / \partial r, \partial M / \partial z)$. The first term on the rhs of Eq. (20) is the overturning response, associated with the linear response due to the change in $\mathbf{U}$ holding the CTRL vortex $(\nabla M)$ constant. The second term is the vortex response, associated with the linear response of $\nabla M$ holding the CTRL overturning (U) constant. The final term is the nonlinear residual, which quickly becomes large as the responses of the primary and secondary circulations both become strong. We focus on the contribution of each term within the boundary layer in the inner core of the storm within the initial 12-h window (Figs. 8, 9).

For both drying and roughening, the total change in $\mathrm{ADV}_{M}$ (Figs. 12a-f) in the inner region boundary layer $(z=0-2 \mathrm{~km} ; r=60-300 \mathrm{~km})$ is primarily controlled by the change in overturning response $\Delta \mathbf{U} \cdot \nabla M$ (Figs. 12g-1). The vortex responses (Figs. 12m-r) to either surface drying or roughening are concentrated near the eyewall $(r=20-60 \mathrm{~km})$ as are the residual responses (Figs. 12s-x), indicating highly nonlinear behavior there associated with shifts in the eyewall. Although the vortex response is large in this region, for drying $\triangle \mathrm{ADV}_{M}$ is nearly uniformly negative and hence is still primarily driven by the overturning response; roughening is more complex and both terms are important. Overall, then, for surface drying, the weakened overturning circulation (Figs. 12g-i) leads to the reduction in $\mathrm{ADV}_{M}$ (Figs. 12a-c), while for roughening, the stronger overturning circulation (Figs. 12j-1) leads to an enhancement of $\mathrm{ADV}_{M}$ (Figs. 12d-f) over the first $12 \mathrm{~h}$. We do not address mechanisms associated with changes in the vortex radial structure here, but this may be an important avenue of future work, particularly 


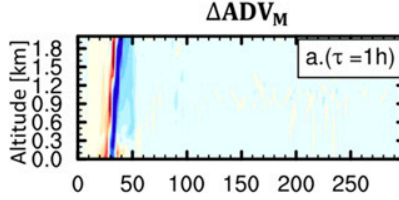

$0.25 \varepsilon$
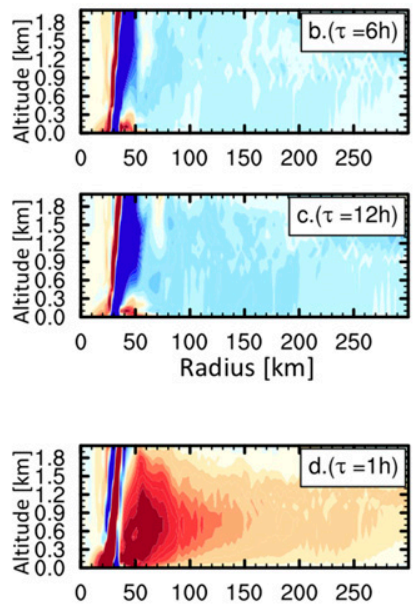

$4 C_{d}$
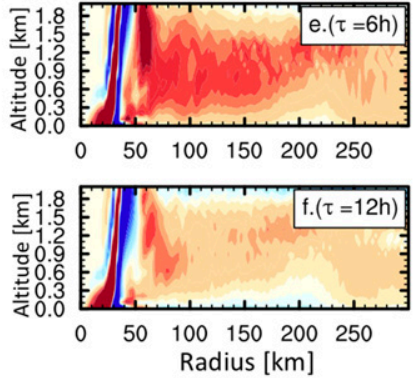

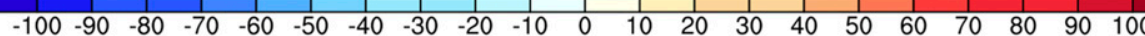
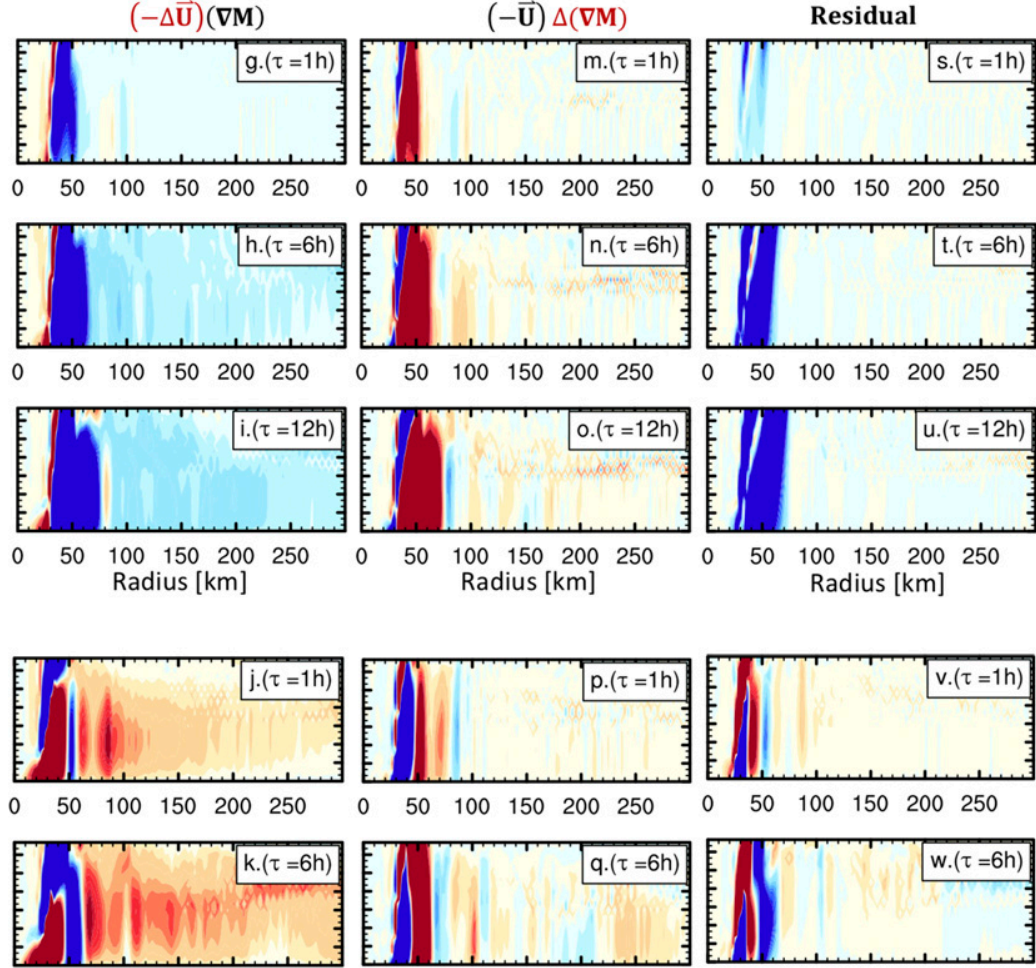

$0 \quad 50 \quad 100 \quad 150 \quad 200 \quad 250$
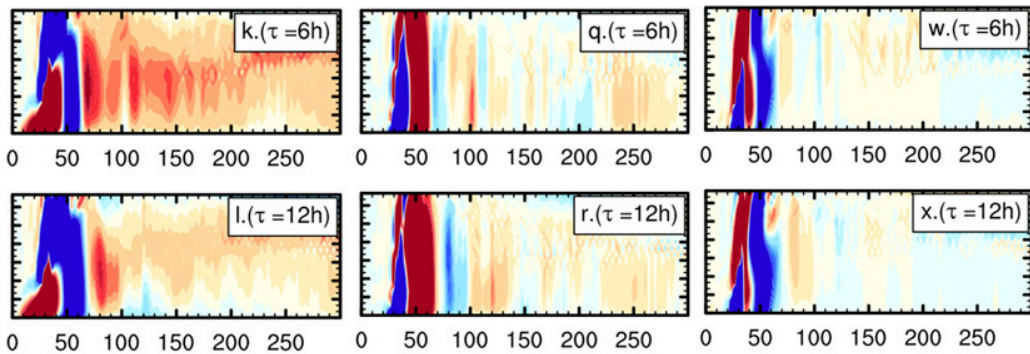

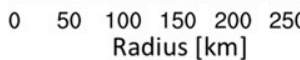

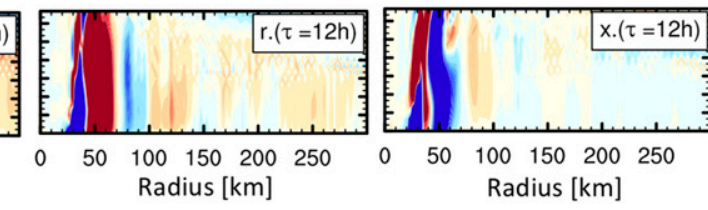

FIG. 12. Linear decomposition of $\Delta \mathrm{ADV}_{M}$ [Eq. (20)] for the first $12 \mathrm{~h}$ of (top) $0.25 \varepsilon$ and (bottom) $4 C_{d}$. Shown are the (a)-(f) total advective $M$ response, $(\mathrm{g})-(\mathrm{l})$ overturning component response, $(\mathrm{m})-(\mathrm{r})$ vortex component response, and (s)-(x) nonlinear residual.

for understanding changes in $r_{\text {max }}$; one simple analysis in this direction is provided in section $5 \mathrm{a}(4)$.

What physical process dominates these contrasting responses of the overturning circulation to each type of surface forcing? The overturning flow response and concurrent thermodynamic response for each experiment are shown in Figs. 13 and 14, respectively.

For surface drying, $\mathrm{ADV}_{M}$ weakens monotonically as the overturning circulation gradually decays (Figs. 13b-e), which starts from the eyewall aloft and reflects the stabilization of the eyewall. This eyewall stabilization process may be quantified via the thermodynamic response of equivalent potential temperature, $\Delta \theta_{e}$ (Fig. 14). Specifically, we define a simple measure of eyewall stability as $\theta_{e, \mathrm{~A}}(\tau)-\theta_{e, \mathrm{~B}}(0)$, where $\mathrm{B}$ denotes the location of maximum vertical velocity in the eyewall at $\tau=0 \mathrm{~h}, \mathrm{~A}$ is the near-surface location beneath $\mathrm{B}$ (A and $\mathrm{B}$ are marked in Fig. 14a). In CTRL, $\theta_{e}$ is nearly conserved moving from
A to $\mathrm{B}$, as would be expected within the strongly convecting eyewall (Fig. 14j). In the drying experiment where low-level wind speeds remain relatively constant by $\tau=6 \mathrm{~h}, \theta_{e}$ decreases in the boundary layer due to the direct reduction of surface moisture availability [Eq. (7); Figs. 14b-d]. The advective response $\Delta\left[-u\left(\partial \theta_{e} / \partial r\right)\right]$ does not contribute to this reduction in $\theta_{e}$, as the advective import of low $\theta_{e}$ air is reduced (which would act to increase $\theta_{e}$ if acting alone) by the gradually weakened overturning circulation (Fig. S2 in the online supplemental material). Because $\theta_{e}$ is reduced specifically within the boundary layer and near the surface beneath the original eyewall, the convective instability of boundary layer parcels near the eyewall is reduced. This boundary layer $\theta_{e}$ reduction gradually stabilizes the eyewall (Fig. 14j), and thus causes the overturning circulation to gradually weaken aloft within the eyewall first (Fig. 14c) and then to extend downward into the boundary layer (Fig. 14d). 

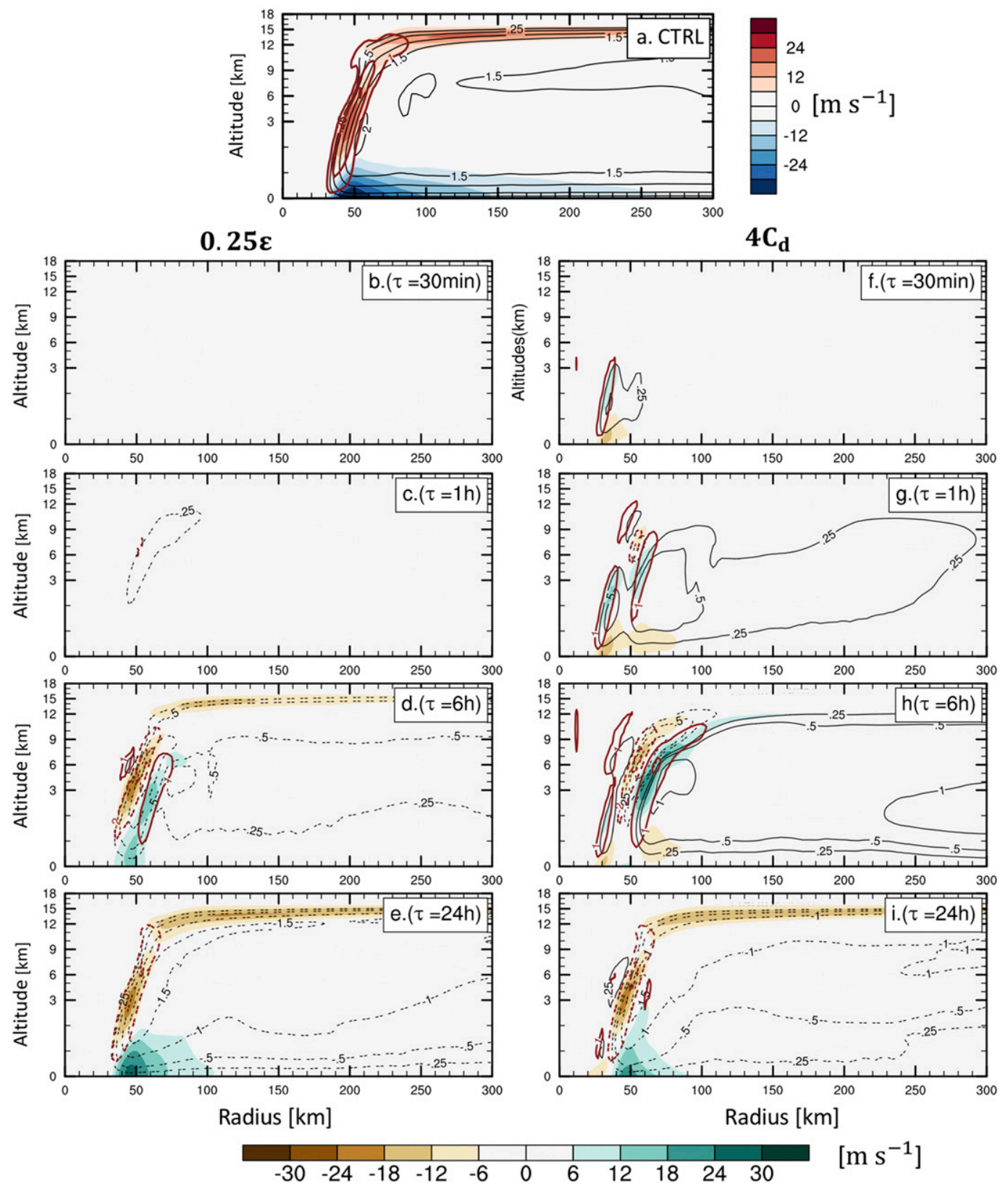

FIG. 13. (a). CTRL mass streamfunction $\psi\left(\left[10^{9} \mathrm{~kg} \mathrm{~s}^{-1}\right.\right.$; black contours), radial wind $u$ (colors) and vertical velocity $w\left(\mathrm{~m} \mathrm{~s}^{-1}\right.$; red contours) at $\tau=1 \mathrm{~h}$. Also shown is the temporal evolution of the responses of mass streamfunction $\Delta \psi$ (solid line for positive response, and dashed line for negative response), radial wind $\Delta u$, and vertical velocity $\Delta w$ for (b)-(e) $0.25 \varepsilon$, and (f)-(i) $4 C_{d}$. For $\tau=30 \mathrm{~min}$ in (b) and (f), the true snapshot is shown rather than the hourly average.

At $\tau=6 \mathrm{~h}$, a slightly stronger updraft forms outside the original eyewall at $r=50 \mathrm{~km}$ while the weakening of the overturning circulation extends through the outflow out to larger radii, with $(\Delta \psi)_{\max } \approx-1.5 \times 10^{9} \mathrm{~kg} \mathrm{~s}^{-1}$ (Fig. 13d). The formation of this new updraft suggests an outward shift of the original eyewall, as boundary layer air, now with a reduced $\theta_{e}$, can only ascend at a larger radius where midlevel $\theta_{e}$ values are initially lower
(Fig. 14a). This is perhaps a simple thermodynamic explanation for why the eyewall and the radius of maximum wind typically expand outwards with weakening. By $\tau=24 \mathrm{~h}$, the temporarily enhanced updraft column gradually weakens, and the overturning circulation continues to weaken throughout the entire vortex (Figs. 13e, 14e). As the overturning circulation continues to weaken, the advective response $\Delta\left[-u\left(\partial \theta_{e} / \partial r\right)\right]$ is increasingly 

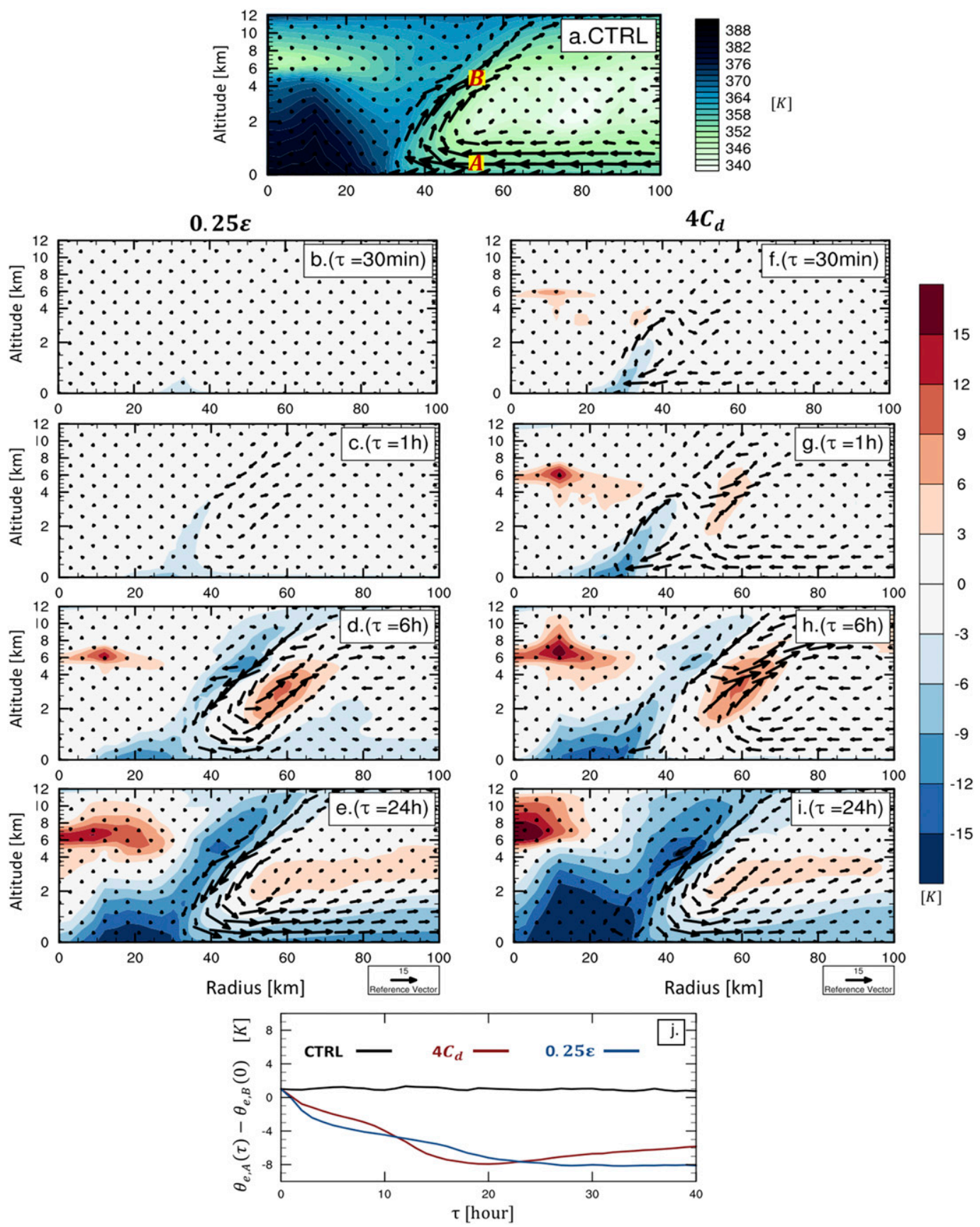

FIG. 14. (a). Equivalent potential temperature $\theta_{e}$ and $r-z$ flow field $\mathbf{U}$ for CTRL at $\tau=1 \mathrm{~h}$. Also shown is the temporal evolution of the response of equivalent potential temperature $\Delta \theta_{e}$ and response of $r-z$ flow field $\Delta \mathbf{U}$ for (b)-(e) $0.25 \varepsilon$ and (f)-(i) $4 C_{d}$. In (a), marker B denotes the location of maximum vertical velocity within the eyewall at $\tau=0 \mathrm{~h}[(r, z)=(54 \mathrm{~km}, 5.24 \mathrm{~km})]$ and marker A denotes the near-surface location beneath $\mathrm{B}[(r, z)=(54 \mathrm{~km}$, $0.05 \mathrm{~km})]$. (j) Temporal evolution of measure of eyewall stability, defined as $\theta_{e, \mathrm{~A}}(\tau)-\theta_{e, \mathrm{~B}}(0)$, for each experiment. Values are calculated as 1 -h averages centered on a given time except for $\tau=30 \mathrm{~min}$, which shows the true snapshot.

positive (i.e., the import of low- $\theta_{e}$ air is reduced), which again indicates that the reduction in $\theta_{e}$ is driven by the reduction in surface fluxes rather than the advective response.
For surface roughening, $\operatorname{ADV}_{M}$ is initially $(\tau \leq 1 \mathrm{~h})$ enhanced by the strengthened near-surface inflow due to Ekman adjustment. An enhanced frictional drag weakens the tangential wind speed and thus weakens the 
outward forces (Coriolis and centrifugal forces), resulting in an inward force imbalance (Anthes 1974) and thus a stronger near-surface inflow (Figs. 13f,g). This stronger inflow first enhances the inner-core overturning circulation, though this frictional enhancement is confined within a relatively shallow layer below $z=4 \mathrm{~km}$ (Fig. 13f). By $\tau=6 \mathrm{~h}$, the overturning circulation is enhanced throughout much of the vortex out to $r=$ $300 \mathrm{~km}$, with two maxima in $\Delta \psi$ and two enhanced updraft columns on either side of the original eyewall (Figs. 13g,h). Though enhanced inflow temporarily enhances inward advection of $M$ during the first $12 \mathrm{~h}$, stronger frictional dissipation rapidly consumes $M$ near the surface. Thus, this strengthened overturning circulation transports reduced- $M$ fluid near the surface inwards and out of the boundary layer, thereby gradually spinning down the vortex aloft through the depth of the troposphere. This overturning circulation and spindown process is analogous to the "stirred tea cup" model in Holton (2004).

While Ekman adjustment rapidly enhances the overturning circulation dynamically, the reduction in surface moisture fluxes associated with the weakened near-surface circulation acts to gradually stabilize the eyewall thermodynamically. The latter eventually dominates, causing the overturning circulation to weaken and hence reduces the inward advection of angular momentum within the boundary layer (Fig. 13i). Relative to CTRL (Fig. 14a), $\theta_{e}$ decreases first within the boundary layer near the eyewall, where the frictionally reduced surface wind speeds reduce $F_{\mathrm{qv}}$ [Eq. (7); Figs. 14f,g]. The advective response $\Delta\left[-u\left(\partial \theta_{e} / \partial r\right)\right]$ also contributes to this initial reduction in $\theta_{e}$ in the first couple of hours, during which the advective import of low $\theta_{e}$ air is reduced very near the surface at the base of the eyewall $(r=30-50 \mathrm{~km})$ but enhanced above this region and at smaller radii just inside of the original eyewall due to the frictionally enhanced overturning circulation (Fig. S3 in the online supplemental material). Similar to the drying experiment, the convective instability of boundary layer parcels near the original eyewall is reduced (Fig. 14j). This effect thermodynamically suppresses the deep overturning through the free troposphere (Figs. 14f,g). Initially, though, this thermodynamic suppression is smaller than the dynamic enhancement induced by surface roughening for $\tau \leq 1 \mathrm{~h}$ below $z=4 \mathrm{~km}$. The competing effect is evident at $\tau=$ $1 \mathrm{~h}$ (Fig. $14 \mathrm{~g}$ ), where $\Delta \mathbf{U}$ is positive below $z=4 \mathrm{~km}$ and negative above $4-\mathrm{km}$ height. Moreover, the response of outward flow at $z=4 \mathrm{~km}$ feeds into a new region of upward motion that begins to emerge outside of the original eyewall at this time. Meanwhile, by $\tau=6 \mathrm{~h}, \theta_{e}$ is strongly reduced near the surface beneath the original eyewall (Fig. 14h), and thus the original eyewall is strongly stabilized. Therefore, the response of the overturning circulation outside the original eyewall is to shift radially outward to where midlevel $\theta_{e}$ values are lower, and hence will be more unstable to boundary layer parcels and thus more conducive to upward motion (Figs. 14a,g,h). However, for surface roughening, the initial Ekman enhancement of the overturning circulation also has a component that extends inside the original eyewall, locally enhancing the updrafts there (Figs. 13f,g). The radius of maximum wind ultimately shifts consistently inwards (Fig. 5c), apparently following the inner updraft. With a weakened overturning circulation, the advective response $\Delta\left[-u\left(\partial \theta_{e} / \partial r\right)\right]$ becomes generally positive (online supplemental Fig. S3), indicating that the reduction in $\theta_{e}$ is driven by the reduction in surface fluxes rather than the advective response, similar to the drying experiment. Finally, boundary layer $\theta_{e}$ continues to decrease and thus become increasingly stable for $\tau=24 \mathrm{~h}$ (Fig. 14i), thereby gradually weakening the deep overturning circulation through the depth of the troposphere within the inner core of the vortex (Fig. 13i).

Overall, while both surface forcings eventually weaken the storm to a similar state, their respective paths to reequilibration are markedly different, particularly during the first $24 \mathrm{~h}$. First, the response of the overturning circulation is directly impacted by decreased surface moisture fluxes, which gradually stabilizes the eyewall updraft. For surface drying, this arises due to the direct reduction of $F_{\mathrm{qv}}$. For surface roughening, $F_{\mathrm{qv}}$ is reduced by the rapidly weakened near-surface wind field, a process that is temporarily amplified by the enhanced advective import of low $\theta_{e}$ air by the Ekman-enhanced radial inflow. This additional, short-term frictional enhancement of the overturning circulation forced by surface roughening can temporarily overcome the effect of eyewall stabilization within the lower troposphere to produce enhanced ascent. This secondary effect also enhances the import of angular momentum, though not enough to fully offset the enhanced loss of angular momentum by surface friction. Thus, the transient response to surface roughening is especially complex, as the primary circulation is directly suppressed dynamically and the secondary circulation is thermodynamically suppressed but dynamically enhanced.

\section{4) Evolution of $r_{\text {MAX }}$}

One particularly thorny yet important quantity is the radius of maximum wind, which lacks a simple governing equation to explain its dynamics. Here we apply our angular momentum response budget framework, which may provide some useful insight into this problem. 

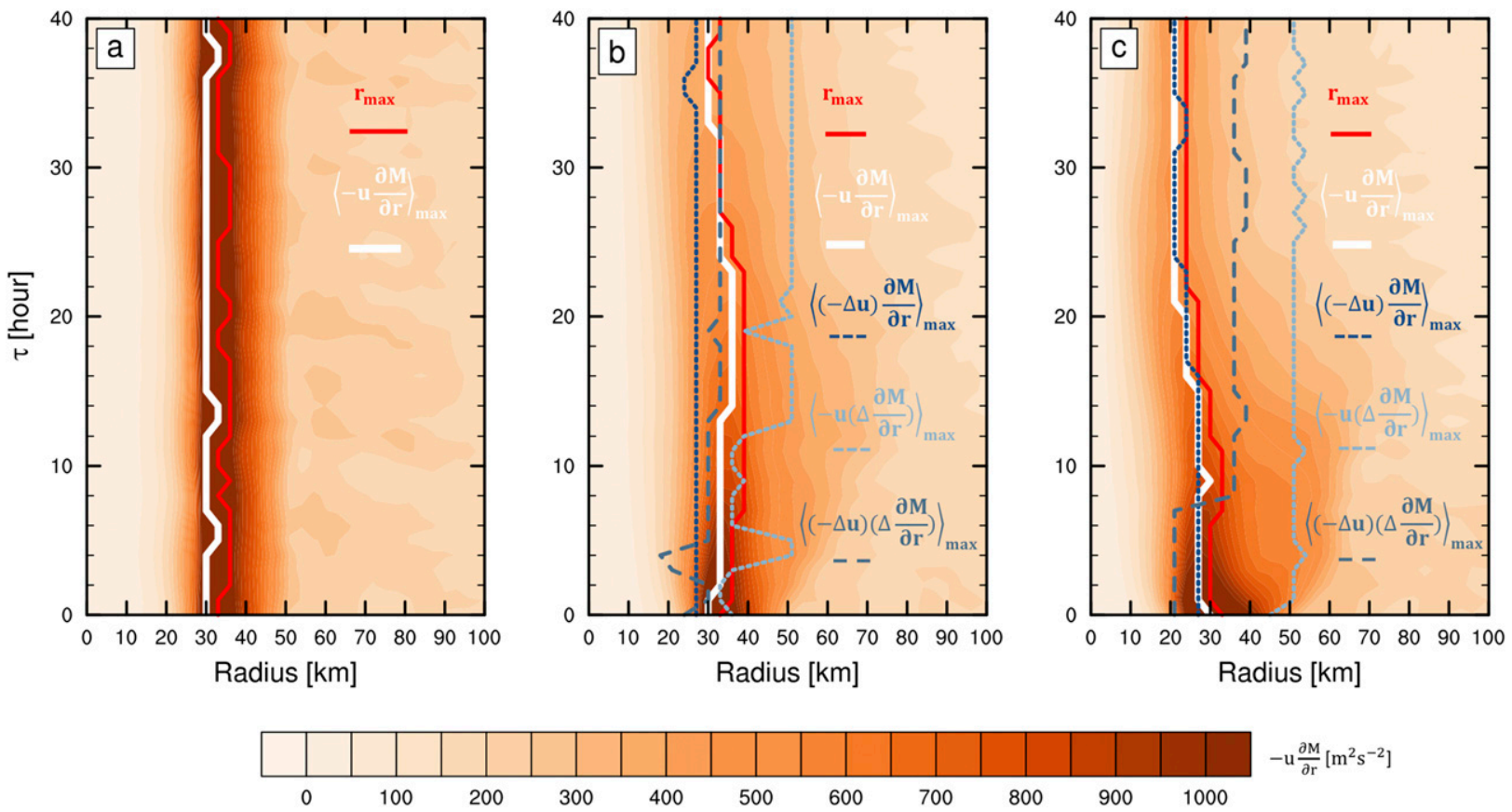

FIG. 15. Hovmöller diagrams of $-u(\partial M / \partial r)$ on the lowest model level (colors) from $\tau=0$ to $40 \mathrm{~h}$, with time series of $r_{\max }$ (red lines) and the radius of maximum $-u\left(\partial M / \partial r\right.$ ) (white lines), for (a) CTRL, (b) $0.25 \varepsilon$, and (c) $4 C_{d}$. Also shown in (b) and (c) are the radii of maximum $(-\Delta u) \partial M / \partial r$ and $-u[\Delta(\partial M / \partial r)]$ and the residuals (blue lines; see legend). Here, \langle\rangle$_{\max }$ denotes radius of maximum value.

Variability in $r_{\max }$ is expected to be intimately linked to horizontal advection of angular momentum, $-u(\partial M / \partial r)$. This advection on the lowest model level is shown in Fig. 15 across CTRL, $0.25 \varepsilon$, and $4 C_{d}$. Notably, the location of maximum $-u(\partial M / \partial r)$ lies just inside of $r_{\max }$ and its temporal variability consistently follows $r_{\max }$ across all three experiments. Thus, analyzing changes in the location of maximum $-u(\partial M / \partial r)$ may offer an avenue for understanding the evolution of $r_{\max }$ using its linear decomposition given by Eq. (20). Specifically, for each term in Eq. (20), we quantify how the radius of its maximum value at the lowest model level evolves in time (Fig. 15, blue lines); \langle\rangle$_{\max }$ denotes this radius. This method provides some insight into how the responses of the overturning circulation and the vortex contribute to the location of $\langle-u(\partial M / \partial r)\rangle_{\max }$. We emphasize that this should not be interpreted as a true budget; it is simply a diagnostic tool for comparing responses due to the overturning circulation and the vortex structure. For surface drying, $\langle-u(\partial M / \partial r)\rangle_{\max }$ lies between $\langle(-\Delta u) \partial$ $M / \partial r)\rangle_{\max }$ and $\langle-u[\Delta(\partial M / \partial r)]\rangle_{\max }$ (Fig. 15b), indicating that neither the overturning response nor vortex structure response is dominant in setting $r_{\max }$. For surface roughening, though, $\langle(-\Delta u) \partial M / \partial r\rangle_{\max }$ closely follows $\langle-u(\partial M / \partial r)\rangle_{\max }$, suggesting a dominant role of the overturning response in setting $r_{\max }$ (Fig. 15c). The above analysis may be valuable for deeper analysis of changes in $r_{\max }$ in future work.

\section{b. Precipitation field}

In addition to the low-level wind field, the precipitation response is also intimately dependent on the response of the secondary circulation in each experiment. To further analyze this relationship, we decompose the response of total precipitation (Fig. 4) into dynamic and thermodynamic components using a simple precipitation scaling, $\tilde{P}$ (Emori and Brown 2005), given by

$$
\tilde{P} \sim \tilde{w} \cdot \tilde{q} .
$$

The dynamical component $\tilde{w}$ is defined as the mean vertical velocity in the volume bounded by $z=1-4 \mathrm{~km}$ and $r=0-150 \mathrm{~km}$, and the thermodynamic component $\tilde{q}$ is defined as the mean 2-m mixing ratio within $r=0-150 \mathrm{~km}$. We may then use Eq. (21) to linearly decompose changes in precipitation (Bony and Emanuel 2001); that is,

$$
\Delta \tilde{P} \approx(\Delta \tilde{w}) \cdot \tilde{q}+\tilde{w} \cdot(\Delta \tilde{q})+(\Delta \tilde{w}) \cdot(\Delta \tilde{q})
$$

which may be written in a normalized form as

$$
\frac{\Delta \tilde{P}}{\tilde{P}_{\mathrm{CTRL}}} \approx \frac{\Delta \tilde{w}}{\tilde{w}_{\mathrm{CTRL}}}+\frac{\Delta \tilde{q}}{\tilde{q}_{\mathrm{CTRL}}}+\frac{(\Delta \tilde{w}) \cdot(\Delta \tilde{q})}{\tilde{P}_{\mathrm{CTRL}}}
$$

where the first term on the rhs of Eq. (23) is the normalized linear response of $\tilde{P}$ due to changes in dynamic component $\tilde{w}$, the second term on the rhs represents the normalized linear response of $\tilde{P}$ due to changes in 

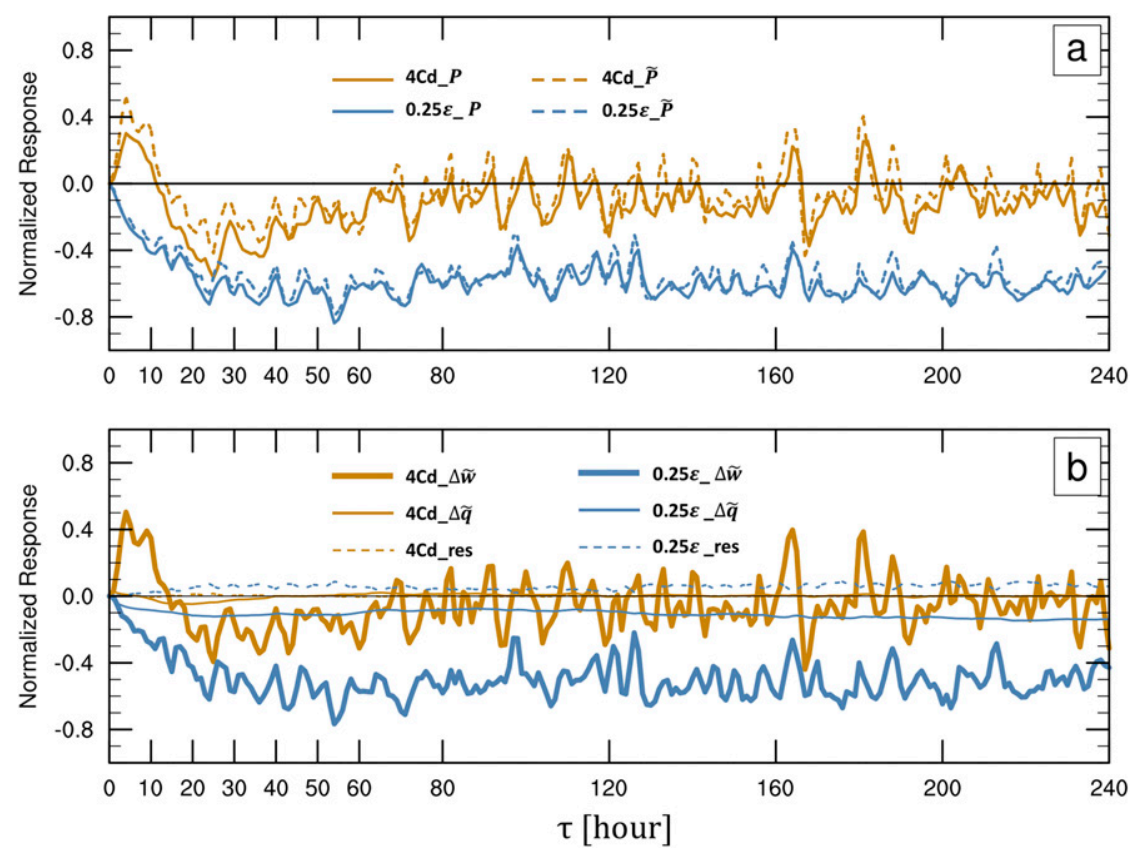

FIG. 16. (a) Temporal evolution of normalized responses in total precipitation $\Delta P / P_{\mathrm{CTRL}}$ and simple decomposition precipitation $\Delta \tilde{P} / \tilde{P}_{\mathrm{CTRL}}$ [Eq. (22)] for $4 C_{d}$ and $0.25 \varepsilon$. (b) Temporal evolution of $\Delta \tilde{P}$ normalized decomposition, including dynamic component $\Delta \tilde{w} / \tilde{w}_{\mathrm{CTRL}}$, thermodynamic component $\Delta \tilde{q} / \tilde{q}_{\text {CTRL }}$, and nonlinear residual [Eq. (23)]. The total hourly precipitation $P$ is integrated within $r=0-150 \mathrm{~km}$. Here, $\tilde{w}$ is the mean vertical velocity between $r=0$ and $150 \mathrm{~km}$ and between $z=1$ and $4 \mathrm{~km}$, representing the dynamic component, and $\tilde{q}$ is the mean 2-m mixing ratio within $r=0-150 \mathrm{~km}$, representing the thermodynamic component. Means and integrals are calculated cylindrically.

thermodynamic component $\tilde{q}$, and the final term is the nonlinear residual. We first compare the normalized responses of model-simulated total precipitation $\Delta P / P_{\mathrm{CTRL}}$ and our simplified precipitation variable $\Delta \tilde{P} / \tilde{P}_{\text {CTRL }}$ for each experiment (Fig. 16a). Indeed, the normalized responses of $\tilde{P}$ closely follow the evolution of $P$.

For surface drying, the reduction in surface fluxes directly weakens the thermodynamic component, with a reduction of $10 \%$ that is relatively constant out to $60 \mathrm{~h}$. Meanwhile, the stabilization of the eyewall updraft causes a significantly stronger dynamic suppression of precipitation, which increases in magnitude quasi exponentially out to $\tau=40 \mathrm{~h}$ (Fig. 16b). This dynamic weakening dominates the decrease in total precipitation throughout the experiment.

For surface roughening, the dynamic response $(\Delta \tilde{w})$ is the dominant control of variability in $\Delta \tilde{P}$ throughout the evolution (Fig. 16b), reaching a maximum enhancement of $+50 \%$ relative to CTRL at $\tau=5 \mathrm{~h}$ and then a maximum reduction of $-40 \%$ relative to CTRL at $\tau=24 \mathrm{~h}$. In contrast, the thermodynamic response $(\Delta \tilde{q})$ remains small and relatively constant throughout the evolution, with only a modest decrease over the first $36 \mathrm{~h}$. This result aligns with the earlier findings that there is an enhancement of the overturning circulation during the first $12 \mathrm{~h}$ for roughening. Thus, the total precipitation remains higher than CTRL in $4 C_{d}$ experiment until $\tau=$ $10 \mathrm{~h}$. Thereafter, the overturning circulation decays as explained in previous section, which weakens the eyewall updraft and directly reduces precipitation.

\section{Conclusions}

Landfalling tropical cyclones produce significant inland hazards, particularly high winds and rainfallinduced flooding. These hazards are intrinsically tied to the response of the TC low-level wind field after landfall. Two key surface forcings associated with the transition from ocean to land are drying and roughening. Here we have tested the response of the wind and precipitation fields in idealized axisymmetric numerical model experiments in which the surface drag coefficient $\left(C_{d}\right)$ or the surface evaporative fraction $(\varepsilon)$ is instantaneously modified over a range of values of each to roughen or dry the surface beneath a mature storm. We characterized the temporal responses of TC intensity, size, and precipitation across experiments and then quantified the physical processes that underlie these 
responses, focusing on one representative experiment from each set that carries similar predicted intensity responses based on potential intensity theory. Key findings are as follows:

1) Surface drying induces a single dominant response whose time scale scales with the magnitude of drying. The primary circulation gradually weakens only within the inner core in response to the gradual eyewall stabilization due to the rapid reduction in surface moisture fluxes. This stabilization progressively weakens the overturning circulation and hence reduces the advective source of angular momentum near the eyewall. This dynamical response in the overturning circulation is the dominant factor in the suppression of precipitation, with additional smaller reductions due to reduced boundary layer moisture. The response of the low-level wind field is initially strongest near $r_{\max }$ and decreases rapidly with radius, such that the outer circulation remains relatively constant; $r_{34 \mathrm{kt}}$ decreases slowly with the weakening storm. The $r_{\max }$ response is nonmonotonic, as it initially increases for weak to moderate drying but decreases with strong drying; all cases eventually reequilibrate to a value slightly smaller than its initial value.

2) Surface roughening induces responses on two distinct time scales. The primary circulation is nearly instantaneously weakened at all radii near the surface due to the direct enhancement of the angular momentum sink from surface friction. Meanwhile, the secondary circulation is temporarily enhanced due to the strengthened frictionally induced inflow. This occurs despite the thermodynamic eyewall stabilization caused by both reduced moisture fluxes associated with the reduced near-surface wind speeds and enhanced advection of low- $\theta_{e}$ air. This strengthened overturning circulation also temporarily increases precipitation within the eyewall region while eventually spinning down the vortex aloft by importing low angular momentum fluid out of the boundary layer. The overturning circulation subsequently weakens after $12 \mathrm{~h}$, as eyewall stabilization gradually weakens the entire overturning circulation similar to surface drying. These responses also act to reduce precipitation back toward its initial value. Storm size $\left(r_{\max }\right.$ and $\left.r_{34 \mathrm{kt}}\right)$ decrease as the storm weakens.

The above findings are summarized schematically in Fig. 17. For surface drying, the feedback between the vortex and surface heat fluxes is weakened directly by reducing $F_{\mathrm{qv}}$, which weakens the vortex slowly by gradually weakening the convective overturning circulation via stabilization of the eyewall that suppresses ascent. Thus, the overturning response exhibits a single time scale throughout the evolution. For surface roughening, the feedback is diminished by reducing $F_{\mathrm{qv}}$ due to the strongly decreased near-surface wind speeds of the vortex associated with enhanced surface friction, which also weakens the convective overturning circulation and further decays the vortex. However, this overturning response emerges following an initial period of frictional enhancement, resulting in a two-stage response to surface roughening. Although they are decaying through different pathways, the long-term transient responses to roughening and drying are similar, leaving a smaller and weaker vortex with a shallower circulation.

Note that the response time scales will differ for real storms in nature since surface properties will change over a finite time scale beneath the storm (and in a heterogeneous manner). The axisymmetric responses presented here may be considered akin to a 3D storm in the limit of a very rapid translation speed from uniform ocean to uniform land. These experiments help us to understand the fundamental underlying responses of the TC circulation to these surface forcings in a simpler setting, which provides the foundation for understanding the more complex response in a real-world setting.

Our findings indicate distinct responses of the wind and precipitation fields to surface roughening and drying on short time scales ( $\sim 0-2$ days) relevant to real-world landfall. This suggests that variations in surface land cover and moisture in the vicinity of storm landfall in the real world may have important effects on the associated inland hazards. Based on the results here, landfall over flat, smooth, dry land, may allow a storm to sustain its circulation for a longer duration despite the strong reduction in surface latent heat fluxes. In contrast, landfall over rough, moist land may rapidly weaken the storm wind field while greatly enhancing rainfall production. This work is a key first step toward understanding the physics of inland TC hazards, which is critical to understanding how inland hazards may change in a future climate state.

We have attempted to characterize and understand the detailed responses to idealized surface roughening and drying, but certain aspects were left for future work. First, the complex response of $r_{\max }$ for different magnitudes of surface drying is not explained here. A deeper analysis of the long-term response evolution beyond $\tau=$ $40 \mathrm{~h}$ may be insightful to understand how the storm responds as the large-scale environment itself gradually evolves. It is unclear why our experiments with frictional enhancement did not produce a transient intensification period, in contrast to past work (Montgomery et al. 2001). Moreover, results may ultimately be sensitive to the details of boundary layer eddies at very fine scales, which are necessarily parameterized in our model. Details of the boundary layer dynamics may require even higher 


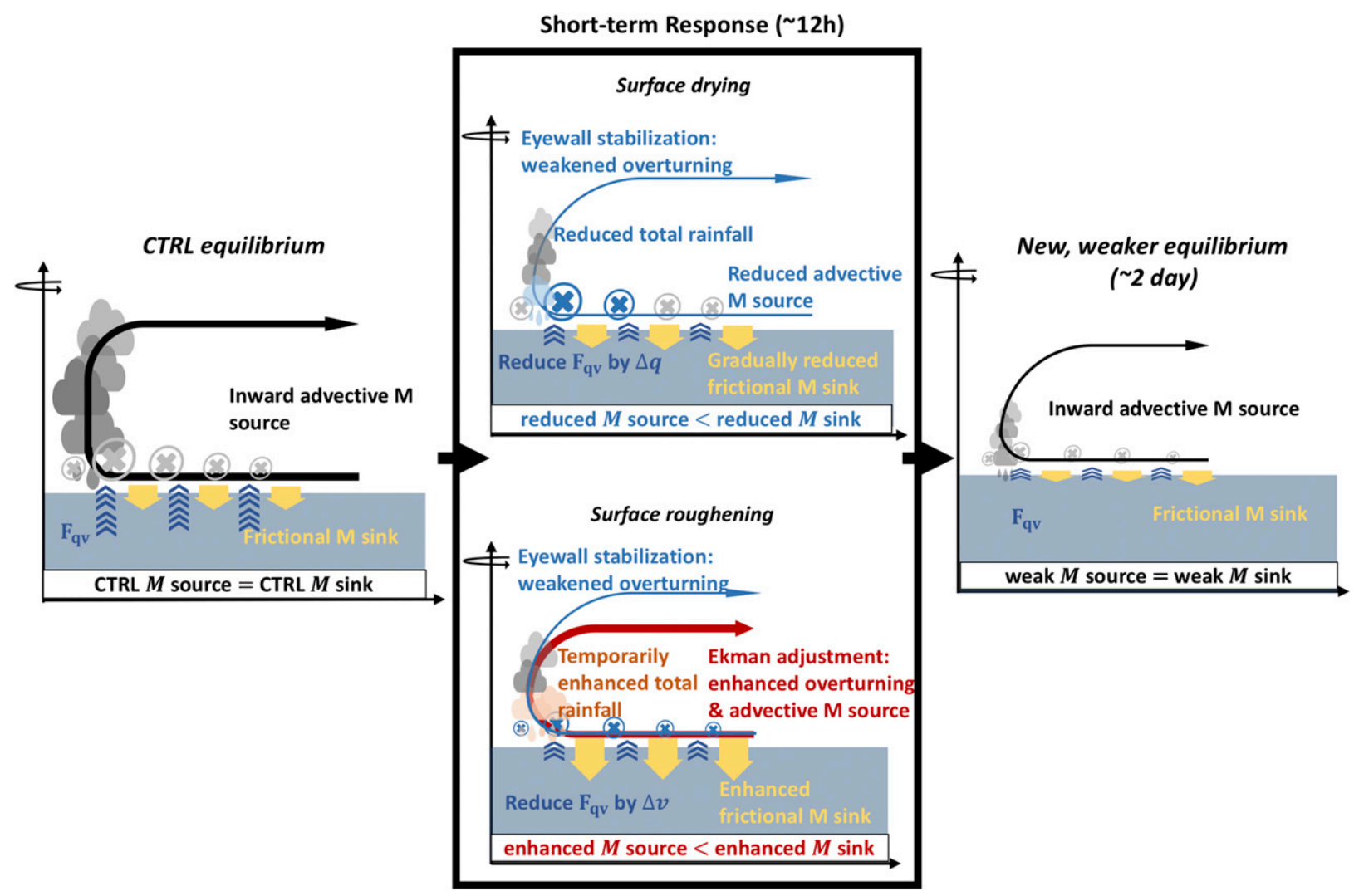

FIG. 17. A conceptual schematic of the short-term response and long-term reequilibration to surface roughening or drying. The schematic shows primary circulation (crosses) and overturning circulation and depicts responses to surface moisture fluxes $F_{\mathrm{qv}}$, angular momentum sources and sinks, and precipitation. The response magnitude of each quantity is indicated by the icon width or size, with an enhancement or reduction colored red or blue, respectively.

resolution to reproduce the finescale structure and evolution of the low-level wind field.

In moving beyond this work, many open questions remain with regard to the processes involved in landfall: what is the response to roughening if $C_{k}$ is allowed to scale with $C_{d}$ ? Can existing theories for intensification, size, and structure explain these wind field responses? How does $C_{k}$ and the ratio of $C_{k} / C_{d}$ vary over the land? How do antecedent soil moisture and storm precipitation affect surface moisture fluxes? How does the size and location of land relative to the storm center alter these responses? How will these axisymmetric responses change when adding real-world complexity, including three-dimensional geometry as well as the translation speed? More generally, the complex real world clearly includes a range of additional types of variability in the transition from ocean to land, including but not limited to subsurface soil properties, ocean upwelling, coastline geometry, and topography, all of which may influence the evolution of low-level wind field and precipitation fields in real storms. In particular, we noted above that sensible heat fluxes may play a more significant role well inland at low intensities (Kieu 2015). Sensible heat fluxes are sensitive to land surface properties and have large diurnal and spatial variation. In our work with fixed surface temperatures, sensible heat fluxes play a minimal role. However, idealized experiments with a coupled land-atmosphere model that allows for land feedbacks may yield different results.

Future work may seek to test the responses in analogous three-dimensional modeling experiments to examine how our axisymmetric results are altered in the presence of resolved azimuthal vortex asymmetries or a finite storm translation speed. Such three-dimensional experiments can serve as a bridge from our axisymmetric results toward a more realistic landfall while still maintaining sufficient simplicity to permit rigorous quantitative analysis and understanding. Comparing these idealized studies with analyses of observational data and high-resolution numerical model outputs would help to link this simple understanding with the real world and to identify what types of complexities are most important for properly modeling inland hazards in nature.

Acknowledgments. The authors are thankful for all conversations related to this research during the $22 \mathrm{nd}$ 

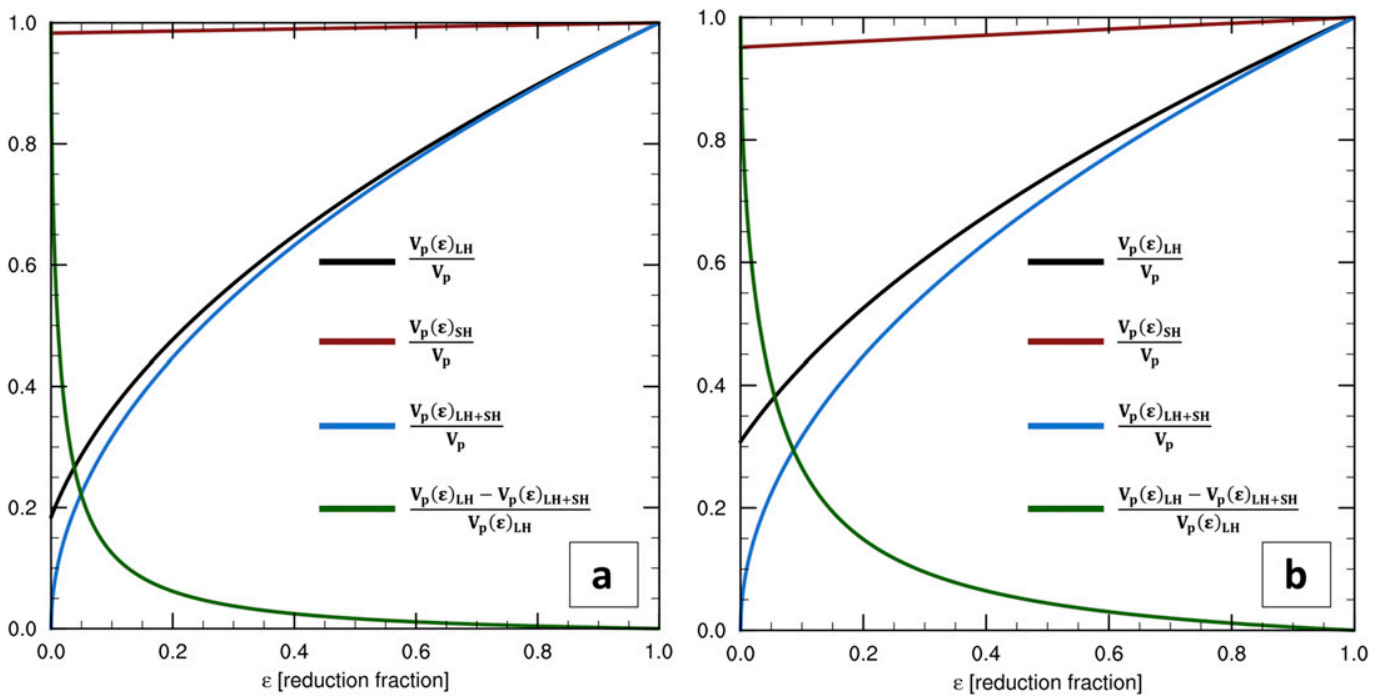

FIG. A1. Ratio of $V_{p}(\varepsilon)_{X} / V_{p}$ with surface flux reduction fraction $\varepsilon$ ranging from 0 to $1 ; X$ denotes the type of surface flux that is modified [latent heat: LH (black curve); sensible heat: SH (red curve); or both (blue curve)]. The ratio $\left[V_{p}(\varepsilon)_{\mathrm{LH}}-V p(\varepsilon)_{\mathrm{LH}+\mathrm{SH}}\right] / V_{p}(\varepsilon)_{\mathrm{LH}}$ is also shown (green curve). Baseline $V_{p}$ is calculated using Eq. (1) from our control using air-sea disequilibrium values from (a) the environment and (b) at $r_{\text {max }}$.

AMS Conference on Atmospheric and Oceanic Fluid Dynamics and 19th Cyclone Workshop. The authors also thankfully acknowledge the helpful feedback from three anonymous reviewers. The authors were supported by U.S. National Science Foundation Grants 1826161 and 1945113 and a Purdue Research Foundation grant.

Data availability statement:. Data for this work are freely available by contacting the corresponding author.

\section{APPENDIX}

\section{Contributions of Surface Sensible and Latent Heat Fluxes to the Potential Intensity}

We may quantify the relative contributions of surface sensible heat fluxes $F_{\mathrm{SH}}$ and latent heat fluxes $F_{\mathrm{LH}}$ to the potential intensity $V_{p}$ for our CTRL simulation. We compare the baseline $V_{p}$ with its value, calculated by assuming that the latent heat fluxes, sensible heat fluxes, or both are reduced by the reduction fraction $\varepsilon$ ranging from 0 to 1 . We denote the latter as $V_{p}(\varepsilon)_{X}$, where $X$ indicates the flux type that is modified. For example, the relative importance of $F_{\mathrm{LH}}$ is given by

$$
\frac{V_{p}(\varepsilon)_{\mathrm{LH}}}{V_{p}}=\frac{\sqrt{\frac{C_{k}}{C_{d}} \eta\left(F_{\mathrm{SH}}+\varepsilon F_{\mathrm{LH}}\right)}}{\sqrt{\frac{C_{k}}{C_{d}} \eta\left(F_{\mathrm{SH}}+F_{\mathrm{LH}}\right)}} .
$$

Figure A1a displays $V_{p}$ calculated using $\varepsilon$ applied only to the latent heat fluxes (black curve), sensible heat fluxes (red curve), and both latent and sensible heat fluxes (blue), each as a fraction of the true $V_{p}$. Figure A1a further displays the fractional contribution of the sensible heat fluxes to $V_{p}$ as $\varepsilon$ is decreased (green curve): [ $V_{p}(\varepsilon)_{\mathrm{LH}}-$ $\left.V p(\varepsilon)_{\mathrm{LH}+\mathrm{SH}}\right] / V_{p}(\varepsilon)_{\mathrm{LH}}$. This ratio indicates that the role of sensible heat fluxes remains small $(<10 \%)$ except when the latent heat fluxes are very strongly reduced to $\varepsilon \leq 0.1$. For this very strong drying, sensible heat fluxes begin to provide a significant fraction of the total surface heat fluxes and thus to the total $V_{p}$. Results are qualitatively similar when calculating $V_{p}$ using $F_{\mathrm{SH}}$ and $F_{\mathrm{LH}}$ at $r_{\max }$ (Fig. A $1 b$ ), although with a stronger contribution from sensible heat fluxes $(\varepsilon<0.4)$. This is because $\Delta T$ in Eq. (1) is significantly larger at $r_{\max }(0.832 \mathrm{~K})$ than in the environment $(0.33 \mathrm{~K})$. The increasing importance of sensible heat fluxes with strong drying highlights that land surface properties, particularly near the eyewall, may become important for weak inland storms, as has been noted in past studies (Evans et al. 2011).

\section{REFERENCES}

Anthes, R., 1974: The dynamics and energetics of mature tropical cyclones. Rev. Geophys. Space. Phys., 12, 495-522, https:// doi.org/10.1029/RG012I003P00495.

Bell, M. M., M. T. Montgomery, and K. A. Emanuel, 2012: Air-sea enthalpy and momentum exchange at major hurricane wind speeds observed during CBLAST. J. Atmos. Sci., 69, 31973222, https://doi.org/10.1175/JAS-D-11-0276.1.

Berg, R., 2009: Tropical cyclone report-Hurricane Ike. National Hurricane Center Rep., 55 pp., https://www.nhc.noaa.gov/ data/tcr/AL092008_Ike.pdf.

Blake, E. S., and D. A. Zelinsky, 2018: Tropical cyclone report-Hurricane Harvey. National Hurricane Center Rep., 
42 pp., https://www.nhc.noaa.gov/data/tcr/AL092017_ Harvey.pdf.

Bony, S., and K. A. Emanuel, 2001: A parameterization of the cloudiness associated with cumulus convection: Evaluation using TOGA COARE data. J. Atmos. Sci., 58, 3158-3183, https://doi.org/10.1175/1520-0469(2001)058<3158:APOTCA $>$ 2.0.CO;2.

Bryan, G. H., 2012: Effects of surface exchange coefficients and turbulence length scales on the intensity and structure of numerically simulated hurricanes. Mon. Wea. Rev., 140, 11251143, https://doi.org/10.1175/MWR-D-11-00231.1.

_- and J. M. Fritsch, 2002: A benchmark simulation for moist nonhydrostatic numerical models. Mon. Wea. Rev., 130, 2917-2928, https://doi.org/10.1175/1520-0493(2002) $130<2917$ :ABSFMN $>2.0$. CO;2.

_- , and R. Rotunno, 2009a: The influence of near-surface, highentropy air in hurricane eyes on maximum hurricane intensity. J. Atmos. Sci., 66, 148-158, https://doi.org/10.1175/ 2008JAS2707.1.

$\longrightarrow$, and $-2009 \mathrm{~b}$ : The maximum intensity of tropical cyclones in axisymmetric numerical model simulations. Mon. Wea. Rev., 137, 1770-1789, https://doi.org/10.1175/2008MWR2709.1.

Cangialosi, J. P., A. S. Latto, and R. Berg, 2018: Tropical cyclone report-Hurricane Irma. National Hurricane Center Rep., 111 pp., https://www.nhc.noaa.gov/data/tcr/AL112017_Irma.pdf.

Chan, J. C. L., K. S. Liu, S. E. Ching, and E. S. T. Lai, 2004: Asymmetric distribution of convection associated with tropical cyclones making landfall along the South China coast. Mon. Wea. Rev., 132, 2410-2420, https://doi.org/10.1175/15200493(2004)132<2410:ADOCAW>2.0.CO;2.

Charney, J. G., and A. Eliassen, 1964: On the growth of the hurricane depression. J. Atmos. Sci., 21, 68-75, https://doi.org/ 10.1175/1520-0469(1964)021<0068:OTGOTH > 2.0.CO;2.

Chavas, D. R., 2017: A simple derivation of tropical cyclone ventilation theory and its application to capped surface entropy fluxes. J. Atmos. Sci., 74, 2989-2996, https://doi.org/10.1175/ JAS-D-17-0061.1.

, and K. A. Emanuel, 2014: Equilibrium tropical cyclone size in an idealized state of axisymmetric radiative-convective equilibrium. J. Atmos. Sci., 71, 1663-1680, https://doi.org/10.1175/ JAS-D-13-0155.1.

_ tropical cyclone wind field. Part II: Wind field variability. J. Atmos. Sci., 73, 3093-3113, https://doi.org/10.1175/JAS-D15-0185.1.

$\_, \ldots$, and K. Emanuel, 2015: A model for the complete radial structure of the tropical cyclone wind field. Part I: Comparison with observed structure. J. Atmos. Sci., 72, 3647-3662, https:// doi.org/10.1175/JAS-D-15-0014.1.

$\longrightarrow, \ldots$, W. Dong, and Y. Lin, 2016: Observed tropical cyclone size revisited. J. Climate, 29, 2923-2939, https://doi.org/10.1175/ JCLI-D-15-0731.1.

Chen, S., J. A. Knaff, and F. D. Marks, 2006: Effects of vertical wind shear and storm motion on tropical cyclone rainfall asymmetries deduced from TRMM. Mon. Wea. Rev., 134, 3190-3208, https://doi.org/10.1175/MWR3245.1.

Cione, J. J., P. G. Black, and S. H. Houston, 2000: Surface observations in the hurricane environment. Mon. Wea. Rev., 128, 1550-1561, https://doi.org/10.1175/1520-0493(2000)128<1550: SOITHE $>2.0 . \mathrm{CO} ; 2$.

Colon, J. A., 1963: On the evolution of the wind field during the life cycle of tropical cyclones. National Hurricane Research Project Rep. 65, 36 pp.
Cook, K., Ed., 2004: Hadley circulation dynamics. The Hadley Circulation: Present, Past, and Future, Advance in Global Change Research, Vol. 21, Springer, 61-83.

Cosby, B. J., G. M. Hornberger, R. B. Clapp, and T. R. Ginn, 1984: A statistical exploration of the relationships of soil moisture characteristics to the physical properties of soils. Water Resour. Res., 20, 682-690, https://doi.org/10.1029/WR020i006p00682.

Cronin, T. W., and D. R. Chavas, 2019: Dry and semidry tropical cyclones. J. Atmos. Sci., 76, 2193-2212, https://doi.org/10.1175/ JAS-D-18-0357.1.

Davis, C. A., and Coauthors, 2008: Prediction of landfalling hurricanes with the Advanced Hurricane WRF Model. Mon. Wea. Rev., 136, 1990-2005, https://doi.org/10.1175/2007MWR2085.1.

Deng, D., N. E. Davidson, L. Hu, K. J. Tory, M. C. N. Hankinson, and S. Gao, 2017: Potential vorticity perspective of vortex structure changes of Tropical Cyclone Bilis (2006) during a heavy rain event following landfall. Mon. Wea. Rev., 145, 1875-1895, https://doi.org/10.1175/MWR-D-16-0276.1.

Eliassen, A., 1971: On the Ekman layer in a circular vortex. J. Meteor. Soc. Japan, 49A, 784-789, https://doi.org/10.2151/ jmsj1965.49A.0_784.

_ - and M. Lystad, 1977: The Ekman layer of a circular vortex: A numerical and theoretical study. Geophys. Norv., 31, 1-16.

Emanuel, K. A., 1986: An air-sea interaction theory for tropical cyclones. Part I: Steady-state maintenance. J. Atmos. Sci., $\mathbf{4 3}$, 585-605, https://doi.org/10.1175/1520-0469(1986)043<0585: AASITF $>2.0 . \mathrm{CO} ; 2$.

- 2004: Tropical cyclone energetics and structure. Atmospheric Turbulence and Mesoscale Meteorology, E. Fedorovich, R. Rotunno, and B. Stevens, Eds., Cambridge University Press, 165-192.

_ 2017: Assessing the present and future probability of Hurricane Harvey's rainfall. Proc. Natl. Acad. Sci. USA, 114, 12 681-12 684, https://doi.org/10.1073/pnas.1716222114.

_ and R. Rotunno, 2011: Self-stratification of tropical cyclone outflow. Part I: Implications for storm structure. J. Atmos. Sci., 68, 2236-2249, https://doi.org/10.1175/JAS-D-10-05024.1.

_ J. Callaghan, and P. Otto, 2008: A hypothesis for the redevelopment of warm-core cyclones over northern Australia. Mon. Wea. Rev., 136, 3863-3872, https://doi.org/10.1175/ 2008MWR2409.1.

Emori, S., and S. J. Brown, 2005: Dynamic and thermodynamic changes in mean and extreme precipitation under changed climate. Geophys. Res. Lett., 32, L17706, https://doi.org/ 10.1029/2005GL023272.

Evans, C., R. S. Schumacher, and T. J. Galarneau, 2011: Sensitivity in the overland reintensification of Tropical Cyclone Erin (2007) to near-surface soil moisture characteristics. Mon. Wea. Rev., 139, 3848-3870, https://doi.org/10.1175/2011MWR3593.1.

Guimond, S. R., M. A. Bourassa, and P. D. Reasor, 2011: A latent heat retrieval and its effects on the intensity and structure change of Hurricane Guillermo (1997). Part I: The algorithm and observations. J. Atmos. Sci., 68, 1549-1567, https://doi.org/ 10.1175/2011JAS3700.1.

Held, I. M., 2005: The gap between simulation and understanding in climate modeling. Bull. Amer. Meteor. Soc., 86, 1609-1614, https://doi.org/10.1175/BAMS-86-11-1609.

Holland, G., J. Belanger, and A. Fritz, 2010: A revised model for radial profiles of hurricane winds. Mon. Wea. Rev., 138, 43934401, https://doi.org/10.1175/2010MWR3317.1.

Holmes, J., Ed., 2001: Wind Loading Structure. Spon Press, 56 pp. Holton, J. R., 2004: An Introduction to Dynamic Meteorology. International Geophysics Series, Vol. 88, Academic Press, 535 pp. 
Hsu, L. H., and H. C. Kuo, 2013: On the geographic asymmetry of typhoon translation speed across the mountainous island of Taiwan. J. Atmos. Sci., 70, 1006-1022, https://doi.org/10.1175/ JAS-D-12-0173.1.

Jian, G. J., and C. C. Wu, 2008: A numerical study of the track deflection of Supertyphoon Haitang (2005) prior to its landfall in Taiwan. Mon. Wea. Rev., 136, 598-615, https://doi.org/ 10.1175/2007MWR2134.1.

Jin, J., N. L. Miller, and N. Schlegel, 2010: Sensitivity study of four land surface schemes in the WRF Model. Adv. Meteor., 2010, 167436, https://doi.org/10.1155/2010/167436.

Kaplan, J., and M. DeMaria, 1995: A simple empirical model for predicting the decay of tropical cyclone winds after landfall. J. Appl. Meteor., 34, 2499-2512, https://doi.org/10.1175/15200450(1995)034<2499:ASEMFP > 2.0.CO;2.

Kepert, J. D., 2012: The impact of landfall on tropical cyclone boundary layer winds. 25th Conf. on Hurricanes and Tropical Meteorology, San Diego, CA, Amer. Meteor. Soc., 8A.1A, https://ams.confex.com/ams/pdfpapers/37219.pdf.

_ 2013: How does the boundary layer contribute to eyewall replacement cycles in axisymmetric tropical cyclones? J. Atmos. Sci., 70, 2808-2830, https://doi.org/10.1175/JAS-D-13-046.1.

Kieu, C., 2015: Hurricane maximum potential intensity equilibrium. Quart. J. Roy. Meteor. Soc., 141, 2471-2480, https:// doi.org/10.1002/qj.2556.

_ , and D. Zhang, 2010: Genesis of Tropical Storm Eugene (2005) from merging vortices associated with ITCZ breakdowns. Part III: Sensitivity to various genesis parameters. J. Atmos. Sci., 67, 1745-1758, https://doi.org/10.1175/2010JAS3227.1.

Kimball, S. K., 2008: Structure and evolution of rainfall in numerically simulated landfalling hurricanes. Mon. Wea. Rev., 136, 3822-3847, https://doi.org/10.1175/2008MWR2304.1.

Kishtawal, C. M., D. Niyogi, A. Kumar, M. L. Bozeman, and O. Kellner, 2012: Sensitivity of inland decay of North Atlantic tropical cyclones to soil parameters. Nat. Hazards, 63, 15271542, https://doi.org/10.1007/s11069-011-0015-2.

Knutson, T., J. Sirutis, M. Zhao, and R. Tuleya, 2015: Global projections of intense tropical cyclone activity for the late twenty-first century from dynamical downscaling of CMIP5/ RCP4.5 scenarios. J. Climate, 28, 7203-7224, https://doi.org/ 10.1175/JCLI-D-15-0129.1.

Kossin, J., 2018: A global slowdown of tropical-cyclone translation speed. Nature, 558, 104-107, https://doi.org/10.1038/s41586018-0158-3.

Kruk, M. C., E. Gibney, D. Levinson, and M. Squires, 2010: A climatology of inland winds from tropical cyclones for the eastern United States. J. Appl. Meteor. Climatol., 49, 15381547, https://doi.org/10.1175/2010JAMC2389.1.

Kuo, H. L., 1965: On formation and intensification of tropical cyclones through latent heat release by cumulus convection. J. Atmos. Sci., 22, 40-63, https://doi.org/10.1175/15200469(1965)022<0040:OFAIOT $>2.0$. CO 2 .

Li, Q., and Y. Duan, 2013: Sensitivity of quasi-periodic outer rainband activity of tropical cyclones to the surface entropy flux. Acta Meteor. Sin., 27, 636-657, https://doi.org/10.1007/s13351-013-0502-3.

Li, Y., K. K. W. Cheung, and J. C. L. Chan, 2014: Numerical study on the development of asymmetric convection and vertical wind shear during tropical cyclone landfall. Quart. J. Roy. Meteor. Soc., 140, 1866-1877, https://doi.org/10.1002/qj.2259.

Lin, N., J. A. Smith, G. Villarini, T. P. Marchok, and M. L. Baeck, 2010: Modeling extreme rainfall, winds, and surge from Hurricane Isabel (2003). Wea. Forecasting, 25, 1342-1361, https://doi.org/10.1175/2010WAF2222349.1.
Liu, M., G. A. Vecchi, J. A. Smith, and H. Murakami, 2018: Projection of landfalling-tropical cyclone rainfall in the eastern United States under anthropogenic warming. J. Climate, 31, 7269-7286, https://doi.org/10.1175/JCLI-D17-0747.1.

Liu, Z., and M. Alexander, 2007: Atmospheric bridge, oceanic tunnel, and global climatic teleconnections. Rev. Geophys., $\mathbf{4 5}$, RG2005, https://doi.org/10.1029/2005RG000172.

Lonfat, M., R. Rogers, T. Marchok, and F. D. Marks, 2007: A parametric model for predicting hurricane rainfall. Mon. Wea. Rev., 135, 3086-3097, https://doi.org/10.1175/MWR3433.1.

Lu, P., N. Lin, K. A. Emanuel, D. Chavas, and J. Smith, 2018: Assessing hurricane rainfall mechanisms using a physics-based model: Hurricanes Isabel (2003) and Irene (2011). J. Atmos. Sci., 75, 2337-2358, https://doi.org/10.1175/JAS-D-17-0264.1.

Malkus, J. S., and H. Riehl, 1960: On the dynamics and energy transformations in steady-state hurricane. Tellus, 12, 1-20, https://doi.org/10.3402/tellusa.v12i1.9351.

Meng, W., and Y. Wang, 2016: A diagnostic study on heavy rainfall induced by Typhoon Utor (2013) in South China: 1. Rainfall asymmetry at landfall. J. Geophys. Res. Atmos., 121, 12 78112 802, https://doi.org/10.1002/2015JD024646.

Merrill, R. T., 1984: A comparison of large and small tropical cyclones. Mon. Wea. Rev., 112, 1408-1418, https://doi.org/10.1175/ 1520-0493(1984)112<1408:ACOLAS > 2.0.CO;2.

Montgomery, M. T., H. D. Snell, and Z. Yang, 2001: Axisymmetric spindown dynamics of hurricane-like vortices. J. Atmos. Sci., 58, 421-435, https://doi.org/10.1175/1520-0469(2001)058<0421: ASDOHL $>2.0 . \mathrm{CO} ; 2$.

Murakami, H., and Coauthors, 2016: Simulation and prediction of category 4 and 5 hurricanes in the high-resolution GFDL HiFLOR coupled climate model. J. Climate, 28, 9058-9079, https://doi.org/10.1175/JCLI-D-15-0216.1.

NCAR, 2019: The NCAR Command Language, version 6.6.2. UCAR-NCAR, http://doi.org/10.5065/D6WD3XH5.

Nolan, D., J. Zhang, and D. Stern, 2009: Evaluation of planetary boundary layer parameterizations in tropical cyclones by comparison of in-situ observations and high-resolution simulations of Hurricane Isabel (2003). Part I: Initialization, maximum winds, and the outer-core boundary layer. Mon. Wea. Rev., 137, 3651-3674, https://doi.org/10.1175/2009MWR2785.1.

Ooyama, K., 1964: A dynamical model for the study of tropical cyclones development. Geofys. Int., 4, 187-198.

- 1969: Numerical simulation of the life cycle of tropical cyclones. J. Atmos. Sci., 26, 3-40, https://doi.org/10.1175/15200469(1969)026<0003:NSOTLC $>2.0 . C O ; 2$.

Peng, K., R. Rotunno, and G. H. Bryan, 2018: Evaluation of a timedependent model for the intensification of tropical cyclones. J. Atmos. Sci., 75, 2125-2138, https://doi.org/10.1175/JAS-D17-0382.1.

Persing, J., and M. T. Montgomery, 2003: Hurricane superintensity. J. Atmos. Sci., 60, 2349-2371, https://doi.org/10.1175/15200469(2003)060<2349:HS>2.0.CO;2.

Powell, D. P., P. P. Dodge, and M. L. Black, 1991: The landfall of Hurricane Hugo in the Carolinas: Surface wind distribution. Wea. Forecasting, 6, 379-399, https://doi.org/10.1175/15200434(1991)006<0379:TLOHHI > 2.0.CO;2.

Powell, M. D., 1982: The transition of the Hurricane Frederic boundary-layer wind field from the open Gulf of Mexico to landfall. Mon. Wea. Rev., 110, 1912-1932, https://doi.org/ 10.1175/1520-0493(1982)110<1912:TTOTHF $>2.0 . C O ; 2$. 1987: Changes in the low-level kinematic and thermodynamic structure of Hurricane Alicia (1983) at landfall. Mon. Wea. 
Rev., 115, 75-99, https://doi.org/10.1175/1520-0493(1987) $115<0075$ :CITLLK $>2.0$. CO 2 .

Rappaport, E., 2000: Loss of life in the United States associated with recent Atlantic tropical cyclones. Bull. Amer. Meteor. Soc., 81, 2065-2073, https://doi.org/10.1175/1520-0477(2000) 081<2065:LOLITU > 2.3.CO;2.

—, 2014: Fatalities in the United States from Atlantic tropical cyclones: New data and interpretation. Bull. Amer. Meteor. Soc., 95, 341-346, https://doi.org/10.1175/BAMS-D-12-00074.1.

Rogers, R., S. S. Chen, J. Tenerelli, and H. Willoughby, 2003: A numerical study of the impact of vertical shear on the distribution of rainfall in Hurricane Bonnie (1998). Mon. Wea. Rev., 131, 1577-1599, https://doi.org/10.1175//2546.1.

Rotunno, R., and G. H. Bryan, 2012: Effects of parameterized diffusion on simulated hurricanes. J. Atmos. Sci., 69, 22842299, https://doi.org/10.1175/JAS-D-11-0204.1.

Rousseau-Rizzi, R., and K. Emanuel, 2019: An evaluation of hurricane superintensity in axisymmetric numerical models. J. Atmos. Sci., 76, 1697-1708, https://doi.org/10.1175/JAS-D-18-0238.1.

Shen, W., I. Ginis, and R. Tuleya, 2001: A numerical investigation of land surface water on landfalling hurricanes. Mon. Wea. Rev., 59, 789-802, https://doi.org/10.1175/1520-0469(2002) 059\%3C0789:ANIOLS\%3E2.0.CO;2.

Sherburn, K., and M. Parker, 2019: The development of severe vortices within simulated high-shear, low-CAPE convection. Mon. Wea. Rev., 147, 2189-2216, https://doi.org/10.1175/MWR-D-18-0246.1.

Smith, R., M. Montgomery, and H. Bui, 2018: Axisymmetric balance dynamics of tropical cyclone intensification and its breakdown revisited. J. Atmos. Sci., 75, 3169-3189, https:// doi.org/10.1175/JAS-D-17-0179.1.

Stull, R. B., 1988: Boundary layer conditions and surface forcings. An Introduction to Boundary Layer Meteorology, R. B. Stull, Ed., Kluwer Academic, 251-294.

Tang, B., and K. Emanuel, 2010: Midlevel ventilation's constraint on tropical cyclone intensity. J. Atmos. Sci., 67, 1817-1830, https://doi.org/10.1175/2010JAS3318.1.

— Bull. Amer. Meteor. Soc., 93, 1901-1912, https://doi.org/ 10.1175/BAMS-D-11-00165.1.

Trapp, R. J., G. R. Marion, and S. W. Nesbitt, 2018: Reply to "Comments on 'The regulation of tornado intensity by updraft width."' J. Atmos. Sci., 75, 4057-4061, https://doi.org/10.1175/ JAS-D-18-0276.1.

Tuleya, R. E., 1994: Tropical storm development and decay: Sensitivity to surface boundary conditions. Mon. Wea.
Rev., 122, 291-304, https://doi.org/10.1175/1520-0493(1994) $122<0291$ :TSDADS $>2.0$.CO;2.

_- and Y. Kurihara, 1975: The energy and angular momentum budgets of a three-dimensional tropical cyclone model. J. Atmos. Sci., 32, 287-301, https://doi.org/10.1175/15200469(1975)032<0287:TEAAMB > 2.0.CO;2.

—, and - 1978: A numerical simulation of the landfall of tropical cyclones. J. Atmos. Sci., 35, 242-257, https://doi.org/ 10.1175/1520-0469(1978)035<0242:ANSOTL>2.0.CO;2.

Villarini, G., R. Goska, J. A. Smith, and G. A. Vecchi, 2014: North Atlantic tropical cyclones and U.S. flooding. Bull. Amer. Meteor. Soc., 95, 1381-1388, https://doi.org/10.1175/BAMS-D13-00060.1.

Willoughby, H. E., 1979: Forced secondary circulations in hurricanes. J. Geophys. Res., 84, 3173-3183, https://doi.org/10.1029/ JC084iC06p03173.

Wing, A. A., and K. A. Emanuel, 2014: Physical mechanisms controlling self-aggregation of convection in idealized numerical modeling simulations. J. Adv. Model. Earth Syst., 6, 59-74, https://doi.org/10.1002/2013MS000269.

_ , S. J. Camargo, and A. H. Sobel, 2016: Role of radiativeconvective feedbacks in spontaneous tropical cyclogenesis in idealized numerical simulations. J. Atmos. Sci., 73, 2633-2642, https://doi.org/10.1175/JAS-D-15-0380.1.

Wong, M. L. M., and J. C. L. Chan, 2007: Modeling the effects of land-sea roughness contrast on tropical cyclone winds. J. Atmos. Sci., 64, 3249-3264, https://doi.org/10.1175/JAS4027.1.

Yang, B., Y. Wang, and B. Wang, 2007: The effect of internally generated inner-core asymmetries on tropical cyclone potential intensity. J. Atmos. Sci., 64, 1165-1188, https://doi.org/ 10.1175/JAS3971.1.

Zhang, F., Z. Pu, and C. Wang, 2017: Effects of boundary layer vertical mixing on the evolution of hurricanes over land. Mon. Wea. Rev., 145, 2343-2361, https://doi.org/10.1175/MWR-D16-0421.1.

,-- , and -2019 : Impacts of soil moisture on the numerical simulation of a post-landfall storm. J. Meteor. Res., $\mathbf{3 3}$, 206-218, https://doi.org/10.1007/s13351-019-8002-8.

Zhang, K., L. Wang, and Y. Zhang, 2016: Improved finite difference method with a compact correction term for solving Poisson's equations. Numer. Heat Transfer, 70B, 393-405, https://doi.org/10.1080/10407790.2016.1215715.

Zhu, L., S. M. Quiring, and K. A. Emanuel, 2013: Estimating tropical cyclone precipitation risk in Texas. Geophys. Res. Lett., 40, 6225-6230, https://doi.org/10.1002/2013GL058284. 